Voix et Images

voixetimages

\title{
Bibliographie de Nicole Brossard
}

\section{Valérie Mailhot et Catherine Parent Beauregard}

Volume 37, numéro 3 (111), printemps-été 2012

Nicole Brossard

URI : https://id.erudit.org/iderudit/1011956ar

DOI : https://doi.org/10.7202/1011956ar

Aller au sommaire du numéro

Éditeur(s)

Université du Québec à Montréal

ISSN

0318-9201 (imprimé)

1705-933X (numérique)

Découvrir la revue

Citer ce document

Mailhot, V. \& Parent Beauregard, C. (2012). Bibliographie de Nicole Brossard.

Voix et Images, 37(3), 97-123. https://doi.org/10.7202/1011956ar d'utilisation que vous pouvez consulter en ligne.

https://apropos.erudit.org/fr/usagers/politique-dutilisation/ 


\title{
B IB LIOGRA PHIE DE NICOLE BROSSARD
}

\author{
$+++$ \\ VALÉRIE MAILHOT \\ Université McGill \\ CATHERINE PARENT BEAUREGARD
}

Université de Montréal

\section{E U VRES}

A. Póésie

+ «Aube à la saison », Michel Beaulieu, Nicole Brossard et Micheline de Jordy, Trois, Montréal, Les Presses de l’A.G.E.U.M., coll. «Cahiers/A.G.E.U.M», 1965, 91 p.

+ Mordre en sa chair, Montréal, Éditions Estérel, 1966, 56 p.

+ L'écho bouge beau, Montréal, Éditions Estérel, coll. "Quoi », 1968, 50 p.

+ Le centre blanc, couverture et illustrations de Marcel Saint-Pierre, Montréal, Éditions d'Orphée, 1970, [n.p.].

+ Suite logique. Poèmes, Montréal, l'Hexagone, 1970, 58 p.

+ Mécanique jongleuse, [s.l.], [s.n.], coll. «Génération», 1973, 20 p. (Réédition: suivi de Masculin grammaticale, Montréal, l'Hexagone, 1974, 92 p.)

+ La partie pour le tout, Montréal, l'Aurore, coll. «Lecture en vélocipède», 1975, 76 p.

+ Le centre blanc. Poèmes 1965-1975, Montréal, l'Hexagone, coll. "Rétrospectives», 1978, 422 p.

+ D'arcs de cycle à la dérive, gravure de Francine Simonin, Saint-Jacques-le-Mineur, Éditions de la Maison, 1979, $4 \mathrm{f}$.

+ Amantes, Montréal, Quinze, coll. «Réelles», 1980, 109 p.

+ Le sens apparent, Paris, Flammarion, coll. «Textes», 1980, 76 p.

+ « Domaine d’écriture», La Nouvelle Barre du jour, no 154, 1985, p. 1-47.

+ L'aviva, Montréal, Nouvelle Barre du jour, 1985, 24 p.

+ Mauve, avec Daphne Marlatt, Montréal/Vancouver, Nouvelle Barre du jour/Writing, coll. «Transformance», 1985, 19 p.

+ Character/Jeu de lettres, avec Daphne Marlatt, Montréal/Vancouver, Nouvelle Barre du jour/Writing, coll. «Transformance», 1986, 19 p.

+ Sous la langue/Under Tongue, édition bilingue, traduit du français par Susanne de LotbinièreHarwood, Montréal/Charlottetown, L’Essentielle/Gynergy Books, 1987, 16 p.

+ À tout regard, Montréal, Bibliothèque québécoise, coll. «Littérature», 1989, 197 p.

+ Installations: avec et sans pronoms, Trois-Rivières/Pantin, Écrits des Forges/Castor astral, 1989, 125 p.

+ Typhon dru, photographies et collages de Christine Davies, Colombes, Collectif Génération, 1990, 13 f. doubles. (Réédition : suivi de La matière harmonieuse manœuvre encore, édition bilingue, traduit du français par Carolyne Bergvall, Londres, Reality Press, 1997, 44 p.)

+ La nuit verte du parc labyrinthe, Laval, Éditions Trois, coll. «Topaze», 1992, 54 p. 
+ Langues obscures, Montréal, l'Hexagone, coll. «L’Hexagone/Poésie», 1992, 59 p.

+ «Flesh, song(e) et promenades», Lèvres urbaines, n 23, 1993, p. 7-27.

+ Vertige de l'avant-scène, Trois-Rivières/Paris, Écrits des Forges/L'Orange bleue, 1997, 84 p.

+ Au présent des veines, Trois-Rivières/Herborn/La Réunion, Écrits des Forges/Éditions Phi/Grand océan, 1999, 135 p.

+ Musée de l'os et de l'eau, Saint-Hippolyte/Saussines, Éditions du Noroît/Cadex Éditions, coll. «Résonance», 1999, 126 p.

+ Cahier de roses \& de civilisation, gravures de Francine Simonin, Trois-Rivières, Éditions d'art Le Sabord, coll. «Excentriq », 2003, 91 p.

+ Je m'en vais à Trieste, Trois-Rivières/Echternach/Limoges, Écrits des Forges/Éditions Phi/Le bruit des autres, 2003, 172 p. (Réédition: Trois-Rivières, Écrits des Forges, 2005, 123 p.)

+ Après les mots, Trois-Rivières/Esch-sur-Alzette, Écrits des Forges/Éditions Phi, 2007, 78 p.

+ Ardeur, Trois-Rivières/Differdange, Écrits des Forges/Éditions Phi, coll. «Graphiti», 2008, 120 p.

+ D'aube et de civilisation. Poèmes choisis, 1965-2007, choix et préface de Louise Dupré, Montréal, TYPO, 2008, 451 p.

+ Lointaines, avec un dessin de Najia Mehadji, Paris, Éditions Caractères, 2010, 45 p.

+ Piano blanc, Montréal, l'Hexagone, coll. «L'appel des mots», 2011, 116 p.

\section{B. Romans et récits}

+ Un livre, Montréal, Éditions du Jour, coll. «Les romanciers du jour», 1970, 99 p. (Réédition: Montréal, Quinze, coll. «Quinze/présence», 1980, 99 p.)

+ Sold-out. Étreinte-illustration, Montréal, Éditions du Jour, coll. «Les romanciers du jour », 1973, 114 p. (Réédition: Montréal, Quinze, coll. «Quinze/présence», 1980, 125 p.)

+ French Kiss. Étreinte-exploration, Montréal, Éditions du Jour, coll. «Nouvelle culture», 1974, 151 p. (Réédition: Montréal, Quinze, coll. «Quinze/présence», 1980, 157 p.)

+ L'amèr ou Le chapitre effrité, Montréal, Quinze, 1977, 99 p. (Réédition: L'amèr ou Le chapitre effrité. Théorie/fiction, Montréal, l'Hexagone, coll. «Typo», 1988, 114 p.)

+ Picture Theory. Théorie-fiction, Montréal, Éditions Nouvelle Optique, coll. «Fiction», 1982, 214 p. (Réédition: Montréal, l'Hexagone, coll. «Typo», 1989, 229 p.)

+ Le désert mauve, Montréal, l'Hexagone, coll. «Fictions», 1987, 220 p.

+ Baroque d'aube, Montréal, l'Hexagone, coll. «Fictions», 1995, 260 p.

+ Hier, Montréal, Québec Amérique, coll. «Mains libres», 2001, 353 p.

+ La capture du sombre, Montréal, Leméac, 2007, 142 p.

\section{Thé âtre}

+ "L'écrivain», Marthe Blackburn, Marie-Claire Blais, Nicole Brossard, Odette Gagnon, Luce Guilbeault, Pol Pelletier et France Théoret, La nef des sorcières, Montréal, Quinze, 1976, 80 p.

\section{Ess a is}

1. Livres

+ Double impression. Poèmes et textes 1967-1984, Montréal, l'Hexagone, coll. «Rétrospectives», $1984,142 \mathrm{p}$.

+ Journal intime ou Voilà donc un manuscrit, Montréal, Les Herbes rouges, 1984, 94 p. (Rééditions: suivi de Euvre de chair et métonymies, Montréal, Les Herbes rouges, 1998, 110 p., et 2008,122 p.) 
+ La lettre aérienne, Montréal, Les Éditions du remue-ménage, coll. «Itinéraires féministes», 1985, $154 \mathrm{p}$.

+ Elle serait la première phrase de mon prochain roman/She Would Be the First Sentence of My Next Novel, édition bilingue, traduit du français par Susanne de Lotbinière-Harwood, Toronto, Mercury Press, 1998, 143 p.

+ L’horizon du fragment, Trois-Pistoles, Éditions Trois-Pistoles, coll. «Écrire», 2004, 130 p.

\section{Parus dans des périodiques et des collectifs}

+ «D’une surface», La Barre du jour, nº 25, été 1970, p. 16-27.

+ «Champ d'action pour figures inédites. Cela s'explique qu'elle manque d'imagination », La Barre du jour, no 29, été 1971, p. 45-53.

+ «Énonciation (sic). Déformation ludique», Stratégie. Pratiques signifiantes, nos 3-4, hiver 1973, p. 51-61.

+ «Vaseline», La Barre du jour, n 42, automne 1973, p. 11-17.

+ «E muet mutant», La Barre du jour, n 50, hiver 1975, p. 10-27.

+ «Car la distance», La Nouvelle Barre du jour, n 58, septembre 1977, p. 5-15.

+ «L'avenir de la littérature québécoise. Aux prises avec la réalité du dedans surgie», Études françaises, vol. XIII, nos 3-4, octobre 1977, p. 383-393.

+ «Le souffle coupé», La Nouvelle Barre du jour, nº 62, janvier 1978, p. 99-104.

+ «Fiction du privé», La Nouvelle Barre du jour, nos 68-69, septembre 1978, p. 67-68.

+ «Les surfaces (Histoire d'écrire)», La Nouvelle Barre du jour, n 81 , septembre 1979, p. 69-80.

+ «La prose maintenant», Ellipse, nos 23-24, 1979, p. 28-30.

+ "L'épreuve de la modernité ou/et les preuves de modernité», La Nouvelle Barre du jour, nos 9091, mai 1980, p. 55-68.

+ «Djuna Barnes: de profil moderne», Mon héroïne. Les lundis de l'histoire des femmes: an 1. Conférences du Théâtre expérimental des femmes, Montréal 1980-81, Montréal, Les Éditions du remue-ménage, coll. «De mémoire de femmes», 1981, p. 189-214.

+ «À la lumière des sens», La Nouvelle Barre du jour, n 112, mars 1982, p. 5-16.

+ «La façon des signes sont des images différées», La Nouvelle Barre du jour, n 140, juin 1984, p. 33.

+ «Exactement là», La Nouvelle Barre du jour, nº 141, septembre 1984, p. 29.

+ «From Radical to Integral», Trivia, n 5, automne 1984 (repris dans La lettre aérienne, puis sous le titre «De radical à intégrales» dans Marisa Zavalloni [dir.], L'émergence d'une culture au féminin, Montréal, Éditions Saint-Martin, coll. «Femmes», 1987, p. 163-174).

+ «Une image captivante», Amazones d'hier, lesbiennes d'aujourd'hui, vol. IV, nº 1, juillet 1985, p. 28-33.

+ «Access to Writing. Ritual of the Written Word», traduit du français par Marian St. Onge et Monique Fol, Trivia, n 8, hiver 1986, p. 8-14.

+ «Le réel et plus», Collectif, Choisir la poésie, Trois-Rivières, Écrits des Forges, 1986 p. 25-26.

+ «Mouvements et stratégies de l'écriture de fiction», Barbara Godard (dir.), Gynocritics. Feminist Approaches to Canadian and Quebec Women's Writing/Gynocritiques. Démarches féministes à l'écriture des Canadiennes et des Québécoises, Toronto, ECW Press, 1987, p. 227-230.

+ " D'être, d'adresse et d'appartenance», Estuaire, no 47, hiver 1987-1988, p. 7-10.

+ «Kind Skin My Mind», Trivia, n 12, printemps 1988, p. 43-44 (texte publié en français sous le titre «Une lesbienne», Treize, vol. I, nº 6, juin-août 1985, p. 17-19). 
+ "Mémoire. Hologramme du désir », La parole métèque, nº 7, 1988, p. 6-8 (repris dans Québec Studies, n 31, printemps-été 2001, p. 8-11, et en anglais, sous le titre «Memory: Hologram of Desire», dans Trivia, no 13, 1988, p. 42-47).

+ "Aura d'une ville», Louise Dupré, Bruno Roy et France Théoret (dir.), Montréal des écrivains. Fiction, Montréal, l'Hexagone, coll. «Typo», 1988, p. 38-42.

+ «L'angle tramé du désir» et «Éperdument», Louky Bersianik, Nicole Brossard, France Théoret, Louise Cotnoir, Gail Scott, Louise Dupré, La théorie, un dimanche, Montréal, Les Éditions du remue-ménage, coll. «Itinéraires féministes», 1988, p. 11-33.

+ "Écriture et homosexualités. Corps de présence», Louis Richard et Marie-Thérèse Séguin (dir.), Homosexualités et tolérance sociale, Moncton, Éditions d'Acadie, 1988, p. 161-165.

+ «Les traces du manifeste», Tessera, vol. VI, printemps 1989, p. VIII-XI.

+ «Mes larmes sont-elles universelles?», Centre québécois du P.E.N. international, L'écrivain: liberté et pouvoir, Sillery, Septentrion, 1989, p. 16-19.

+ «La version des femmes du réel», Cécile Cloutier et Ben Shek (dir.), La poésie de l'Hexagone. Évolution, signification, rayonnement, Montréal, l'Hexagone, 1990, p. 73-77.

+ « Poetic Politics», Charles Bernstein (dir.), The Politics of Poetic Form. Poetry and Public Policy, New York, Roof Books, 1990, p. 73-86.

+ «Femme à la bouche ouverte pendant un bombardement», Arcade, n 25, automne 1992, p. 7.

+ «Ludique, critique et moderne - rebelle/scribelle», Louise Forsyth, Kathleen Kells et Raija Koski (dir.), Les discours féminins dans la littérature postmoderne au Québec, San Francisco, EMText, 1993, p. 107-111.

+ «Procession d'un oui qui va son énergie», Collectif, Mises en scène d'écrivains. Assia Djebar, Nicole Brossard, Madeleine Gagnon, France Théoret, Sainte-Foy/Grenoble, Le Griffon d'argile/Presses universitaires de Grenoble, coll. «Trait d'union», 1993, p. 63-71.

+ "Certain Words», traduit du français par Barbara Godard, Barbara Godard (dir.), Collaboration in the Feminine. Writings on Women and Culture from Tessera, Toronto, Second Story Press, 1994, p. 47-52.

+ «Écrire la société. D'une dérive à la limite du réel et du fictif », Philosophiques, vol. XXI, nº 2, automne 1994, p. 303-320.

+ «Le corps du personnage», Tessera, vol. XIX, hiver 1995, p. 63-71.

+ «Euvre de chair et métonymies», Collectif, Femmes, corps et âme, Québec/Montréal, Musée de la civilisation/XYZ éditeur, 1996, p. 39-52 (repris dans Journal intime suivi de Fuvre de chair et métonymies).

+ «Fluid Arguments», Peter Baker (dir.), Onward: Contemporary Poetry and Poetics, New York, Peter Lang, 1996, p. 315-346.

+ «Écriture lesbienne: stratégie de marque», Didier Eribon (dir.), Les études gay et lesbiennes, Paris, Éditions du Centre Pompidou, coll. «Supplémentaires», 1998, p. 51-56.

+ «Vingt pages entrecoupées de silence», Les Écrits, n 95, 1999, p. 117-140.

+ "A State of Mind in the Garden", Journal of Lesbian Studies, vol. IV, n 4, décembre 2000, p. 35-40.

+ «Le mot de l'Académie - Silence», Le Devoir, 29 juillet 2002, p. A1 et A8.

\section{E. Témoignages, textes d'opinion et conférences}

+ «Une question, des réponses (1)», Liberté, vol. IX, nº 6, novembre-décembre 1967, p. 30-38 (réponse à une enquête).

+ «Les inédits - Saint-Denys Garneau », La Barre du jour, n² 21, septembre-octobre 1969, p. 69. 
+ «Grand risque», Le Devoir, 14 novembre 1969, p. 9.

+ « Naissance et dispersion du désir», Liberté, vol. XIV, nº 6, décembre 1972, p. 20-23.

+ «Ville Saint-Laurent... (décibels)», Le Devoir, supplément littéraire, 18 mai 1974, p. XIX.

+ «Réponses de toutes parts», Le Soleil, 12 octobre 1974, p. D6.

+ «L'interdit posé sur le féminin », Le Devoir, 10 mai 1975, p. 17.

+ «À notre sujet», Chroniques, nº 18-19, juin-juillet 1976, p. 7-9.

+ «La tendance», Liberté, vol. XIX, n 3, mai-juin 1977, p. 41-45 (réponse à une enquête sur la littérature québécoise).

+ «L'opportunité d'un débat de fond sur l'avortement», Le Devoir, 14 octobre 1977, p. 5 (réponse à René Lévesque publiée par Francine Beauchamp, Nicole Brossard, Madeleine Howard-Egre, Michèle Jean et Eliette Rioux).

+ «La question de l'identité», Le Devoir, 3 juin 1978, p. 25.

+ «Traverser la fiction», Le Devoir, 3 juin 1978, p. 36.

+ «Poésie engagée», Revue de l'Université Laurentienne, vol. X, n² 2, 1978, p. 121-125.

+ «Ça fait jaser», Sorcières, no 14, 1978, p. 23-24.

+ «Témoignage», Élisabeth J. Lacelle (dir.), La femme et la religion au Canada français: un fait socio-culturel. Perspectives et prospectives, Montréal, Éditions Bellarmin, coll. «Femmes et religions », 1979, p. 129-132.

+ «Un corps pour écrire», Le Devoir, 24 novembre 1979, p. III.

+ «Fragments d'urgence», Le Devoir, 19 avril 1980, p. 21-22. (Réédition: Yolande Cohen [dir.], Femmes et politique, Montréal, Éditions du Jour, coll. «Les idées du jour», 1981, p. 15-19.)

+ "La réponse des "directrices"; courrier par Nicole Brossard et Andrée Yanacopoulo », Le Devoir, 16 février 1980, p. 31.

+ «Féminisme ou lutte spécifique des femmes », collectif, Les têtes de pioche (1976-1979), Montréal, Les Éditions du remue-ménage, 1980, p. 16 (d'abord publié dans Les Têtes de pioche. Journal des femmes, $\mathrm{n}^{\circ} 1$, mars 1976, p. 4).

+ «La vie privée est politique», collectif, Les têtes de pioches (1976-1979), Montréal, Les Éditions du remue-ménage, 1980, p. 21 (d'abord publié dans Les Têtes de pioche. Journal des femmes, $\mathrm{n}^{\circ}$ 2, avril 1976, p. 1-2).

+ «Les belles-mères», collectif, Les têtes de pioches (1976-1979), Montréal, Les Éditions du remue-ménage, 1980, p. 30 (d'abord publié dans Les Têtes de pioche. Journal des femmes, nº 3 , mai 1976, p. 2).

+ «Le fantasme ou la vraie vie», collectif, Les têtes de pioches (1976-1979), Montréal, Les Éditions du remue-ménage, 1980, p. 39 (d'abord publié dans Les Têtes de pioche. Journal des femmes, no 4 , juin 1976, p. 3).

+ «Première séance: (4 octobre 1975-21 heures)», Liberté, vol. XVIII, nºs 4-5, juilletoctobre 1976, p. 10-13.

+ «Êtes-vous normale? Vos filles seront-elles normales?», collectif, Les têtes de pioches (19761979), Montréal, Les Éditions du remue-ménage, 1980, p. 46 (d'abord publié dans Les Têtes de pioche. Journal des femmes, $n^{\circ} 5$, septembre 1976, p. 2).

+ «Un mouvement de femmes?», collectif, Les têtes de pioches (1976-1979), Montréal, Les Éditions du remue-ménage, 1980, p. 46 (d'abord publié dans Les Têtes de pioche. Journal des femmes, n5, septembre 1976, p. 2).

+ "Dossier: les femmes et le P.Q. Ce que le Parti québécois promet aux femmes», en collaboration avec Michèle Jean, collectif, Les têtes de pioches (1976-1979), Montréal, Les Éditions du 
remue-ménage, 1980, p. 73 et 76 (d'abord publié dans Les Têtes de pioche. Journal des femmes, $n^{\circ} 8$, décembre 1976, p. 5 et 8).

+ « Je suis née fille», collectif, Les têtes de pioches (1976-1979), Montréal, Les Éditions du remueménage, 1980, p. 89 (d'abord publié dans Les Têtes de pioche. Journal des femmes, vol. II, nº 1, mars 1977, p. 5).

+ «Le pétrin, le pétrin», collectif, Les têtes de pioches (1976-1979), Montréal, Les Éditions du remue-ménage, 1980, p. 89 (d'abord publié dans Les Têtes de pioche. Journal des femmes, vol. II, $\mathrm{n}^{\circ} 1$, mars 1977, p. 5).

+ «De l'espace», collectif, Les têtes de pioches (1976-1979), Montréal, Les Éditions du remueménage, 1980, p. 123 (d'abord publié dans Les Têtes de pioche. Journal des femmes, vol. II, nº 5 , septembre 1977, p. 7).

+ « Nicole Brossard», communication présentée à la Rencontre québécoise internationale des écrivains, Liberté, vol. XX, nos 4-5, juillet-octobre 1978, p. 101-104.

+ "Libération de la femme/Mouvement des femmes", collectif, Les têtes de pioches (1976-1979), Montréal, Les Éditions du remue-ménage, 1980, p. 150 (d'abord publié dans Les Têtes de pioche. Journal des femmes, vol. II, no 9-vol. III, nº 1, février-mars 1978, p. 2).

+ « Notes pour une analyse des groupes ou Caroline et Carole», collectif, Les têtes de pioches (1976-1979), Montréal, Les Éditions du remue-ménage, 1980, p. 156 (d'abord publié dans Les Têtes de pioche. Journal des femmes, vol. II, nº 9-vol. III, nº 1, février-mars 1978, p. 8).

+ "L'identité comme science-fiction de soi », Pierre Tap (dir.), Identités collectives et changements sociaux, t. II, Toulouse, Privat, 1981, p. 391-395 (repris dans Vlasta, n 1, printemps 1983, p. 9399).

+ "Conférence des femmes-écrivains en Amérique. Séance inaugurale», Revue de l'Université d'Ottawa, vol. L, no 1, 1981, p. 7-8.

+ "Savoir se synchroniser», La Presse, 17 avril 1982, p. IV.

+ «Pourquoi j'écris», Québec français, n 47, octobre 1982, p. 31.

+ «La conscience à vif», La vie en rose, ñ 11, mai 1983, p. 58.

+ «Tout revoir au féminin», Le Devoir, 25 octobre 1983, p. 6.

+ «La page du livre», Women and Words. The Anthology/Les femmes et les mots. Une anthologie, Madeira Park, Harbour Publishing Company, 1984, p. 126-129.

+ "Recto verso », Irène Belleau et Gilles Dorion (dir.), Les œuvres de création et le français au Québec. Actes du Congrès Langue et société au Québec, Montréal, Éditeur Officiel, 1984, p. 240245.

+ «Tender Skin My Mind», Ann Dybikowski, Victoria Freeman, Daphne Marlatt, Barbara Purling et Betsy Warlord (dir.), In the Feminine. Women and Words/Les femmes et les mots. Conference Proceedings 1983, Edmonton, Longspoon, 1985, p. 180-183.

+ «Un féminisme de préférence», La vie en rose, nº 24, mars 1985, p. 28-29 et 56.

+ «Stricto sensu, attraction», Possibles, vol. XI, n³, printemps-été 1987, p. 174-175.

+ «Les pas juxtaposés du désir», Tessera, vol. V, septembre 1988, p. 29-30.

+ «Le tueur n'était pas un jeune homme», Louise Malette et Marie Chalouh (dir.), Polytechnique, 6 décembre, Montréal, Les Éditions du remue-ménage, coll. «Itinéraires féministes», 1990, p. 29-30 (reprise d'un texte initialement paru dans La Presse, le 21 décembre 1989).

+ «Correspondance, 1986» [avec Michèle Causse], traduit du français par Lise Weil, Trivia, vol. XIX, printemps 1992, p. 52-69.

+ «Les défis de la traduction », Lesbia Magazine, n 101, janvier 1992, p. 24-25. 
+ "I Like to Say We and Look Elsewhere», traduit du français par Pierre Joris, Boundary 2. An International Journal of Literature and Culture, vol. XXVI, n 1, 1999, p. 60-62.

+ «Écrivaine, 19/08/97», Peter Schulman et Mischa Zabotin (dir.), Le dernier livre du siècle. Deux Américains enquêtent sur l'intelligentsia française au tournant du siècle, Paris, Romillat, coll. «Enquête», 2001, p. 252-256.

+ "Quel livre vous redonne espoir en l'humanité ?", réponse à un questionnaire du guide culturel Voir, semaine du 8 au 14 novembre 2001, p. 36-39.

+ «L'ivresse du livre», Danielle Schaub (dir.), Reading Writers Reading. Canadian's Authors Reflections, Edmonton/Jérusalem, University of Alberta Press/Hebrew University Magnes Press, 2006, p. 86-87.

\section{F. Entretiens}

+ Partage du jour, émission radiophonique avec Nicole Brossard et Marcel Saint-Pierre réalisée par Marcel Tremblay, animée par Henri Bergeron et diffusée le 19 février 1965, Société RadioCanada, Centre d'archives Gaston-Miron, http://www.crlq.umontreal.ca/CAGM/rechsimple? query $=55382216$.

+ Carnet arts et lettres, entrevue radiophonique avec Nicole Brossard réalisée par Gilbert Picard, animée par Jean Sarrazin et diffusée le 17 septembre 1970, Société Radio-Canada, Centre d'archives Gaston-Miron, http://www.crlq.umontreal.ca/CAGM/rechsimple?query=586372.

+ Horizons, entrevue radiophonique avec Nicole Brossard réalisée par Fernand Ouellette et diffusée le 24 septembre 1970, Société Radio-Canada, Centre d'archives Gaston-Miron, http://www.crlq.umontreal.ca/CAGM/rechsimple?query=586560.

+ Carnet arts et lettres, entrevue radiophonique avec Nicole Brossard réalisée par Gilbert Picard, animée par Jean Sarrazin et diffusée le 8 décembre 1970, Société Radio-Canada, Centre d'archives Gaston-Miron, http://www.crlq.umontreal.ca/CAGM/rechsimple?query=565589.

+ Horizons, entrevue radiophonique avec Nicole Brossard réalisée par Fernand Ouellette, animée par Jacques Godbout et diffusée le 10 décembre 1970, Société Radio-Canada, Centre d'archives Gaston-Miron, http://www.crlq.umontreal.ca/CAGM/rechsimple?query=565632.

+ La littérature en été, entrevue radiophonique avec Nicole Brossard réalisée par Jacques Thibodeau, animée par Réginald Martel et diffusée le 2 août 1971, Société Radio-Canada, Centre d'archives Gaston-Miron, http://www.crlq.umontreal.ca/CAGM/rechsimple?query=587357.

+ Book-club, émission radiophonique avec Nicole Brossard réalisée par Gilles Archambault, animée par Réginald Martel et diffusée le 6 septembre 1972, Société Radio-Canada, Centre d'archives Gaston-Miron, http://www.crlq.umontreal.ca/CAGM/rechsimple?query=604095.

+ Book-club, entrevue radiophonique avec Nicole Brossard réalisée par Gilles Archambault et Gilles Moreau et diffusée le 12 mars 1973, Société Radio-Canada, Centre d'archives GastonMiron, http://www.crlq.umontreal.ca/CAGM/rechsimple?query=608811.

+ BEAUSOLEIL, Claude et André ROY, «Entretien avec Nicole Brossard », Hobo/Québec, ns 14-15, janvier 1974, p. 12-22.

+ Femme d'aujourd'hui, émission télévisuelle avec Nicole Brossard réalisée par Normand Mathon et Robert Séguin, animée par France Nadeau et diffusée le 16 mai 1975, Société Radio-Canada, Centre d'archives Gaston-Miron, http://www.crlq.umontreal.ca/CAGM/rechsimple?query=12147.

+ La feuillaison, émission radiophonique avec Nicole Brossard réalisée par Gérard Binet, animée par Jacques Fontaine et diffusée le $1^{\text {er }}$ août 1975, Société Radio-Canada, Centre d'archives Gaston-Miron, http://www.crlq.umontreal.ca/CAGM/rechsimple?query=624246. 
+ VAN SCHENDEL, Michel et Jean FISETTE, «Un livre à venir. Rencontre avec Nicole Brossard», Voix et Images du pays, vol. III, no 1, septembre 1977, p. 3-18.

+ Au fil des arts, entrevue radiophonique avec Nicole Brossard réalisée par Raymond Fafard, animée par Marcel Godin et diffusée le 16 septembre 1977, Société Radio-Canada, Centre d'archives Gaston-Miron, http://www.crlq.umontreal.ca/CAGM/rechsimple?query=635823.

+ ROY, Monique, "Écrire "je suis une femme" est plein de conséquences. Entretien avec Nicole Brossard», Le Devoir, 12 novembre 1977, p. 37.

+ BAYARD, Caroline, «Entrevue avec Nicole Brossard», Caroline Bayard et Jack David, Outposts/Avant-postes. Three Solitudes, Contemporary Literary Criticism in Canada, Toronto, Presses Porcépic, 1978, p. 57-74. (Un extrait de l'entrevue est également paru dans Lettres québécoises, $\mathrm{n}^{\circ} 4$, novembre 1976, p. 34-37)

+ Femme d'aujourd'hui, entrevue télévisuelle avec Nicole Brossard réalisée par Yvette Pard, animée par Lise Gauvin et diffusée le 11 octobre 1978, Société Radio-Canada, Centre d'archives Gaston-Miron, http://www.crlq.umontreal.ca/CAGM/rechsimple?query=37550.

+ ROYER, Jean, «Nicole Brossard. La traversée des inédits», Le Devoir, 16 décembre 1978, p. 25.

+ ROY, André, «La fiction vive. Entretien avec Nicole Brossard sur sa prose», Journal of Canadian fiction, ${ }^{\text {os }}$ 25-26, 1979, p. 31-40.

+ DESROSIERS, Diane, «Entrevue avec Nicole Brossard», Versance, vol. I, nº 1, avril 1979, p. 4447.

+ WILSON, Jean, « Nicole Brossard. Fantasies and Realities», Broadside. A Feminist Review, vol. II, $\mathrm{n}^{\circ}$ 8, juin 1981, p. 11-18.

+ MOISAN, Lise, "Nicole Brossard et Adrienne Rich. Conscience lesbienne et littérature », La vie en rose, $\mathrm{n}^{\circ}$ 3, septembre-novembre 1981, p. 50-51.

+ Sans maquillage, émission radiophonique avec Nicole Brossard réalisée par Berthelet Charron, animée par Gérard Leblanc, non diffusée et produite le 29 janvier 1982, Société Radio-Canada, Centre d'archives Gaston-Miron, http://www.crlq.umontreal.ca/CAGM/rechsimple?query= 60039408001.

+ ROYER, Jean «Nicole Brossard. La tentation du roman », Le Devoir, 30 octobre 1982, p. 17-18 (repris sous le titre «La tentation du roman", Les deux rives, n 1 , printemps-été 1984, p. 1112).

+ BEAUSOLEIL, Claude, Hugues CORRIVEAU, Louise COTNOIR et Lise GUẼVREMONT, «Entretien avec Nicole Brossard sur Picture Theory», La Nouvelle Barre du jour, nos 118-119, novembre 1982, p. 177-201.

+ ROY, André, "Écriture et traces lesbiennes», Sortie, n² 2, novembre 1982, p. 3-5.

+ Littérature au pluriel, émission radiophonique avec Nicole Brossard réalisée par Gilles Archambault, animée par Wilfrid Lemoine et diffusée le 9 novembre 1982, Société RadioCanada, Centre d'archives Gaston-Miron, http://www.crlq.umontreal.ca/CAGM/rechsimple? query $=243440001$.

+ GAUDET, Gérald, Nicole Brossard, cassette audio, Trois-Rivières, CFCQ-FM, 1983, 60 min.

+ ROYER, Jean, «Entretien avec Nicole Brossard», Écrivains contemporains. Entretiens, t. II, Montréal, l'Hexagone, 1983, p. 22-31.

+ BEAUSOLEIL, Claude, Hugues CORRIVEAU, Louise COTNOIR et Lise GUẼVREMONT, «Interview with Nicole Brossard on Picture Theory », Canadian Fiction Magazine, n 47, 1983, p. 122-135.

+ TRITON, Suzette, «Rencontre avec Nicole Brossard», Vlasta, nº 1, printemps 1983, p. 33-39. 
+ Impacts, émission télévisuelle avec Nicole Brossard réalisée par André Simard, animée par Robert-Guy Scully et diffusée le 14 avril 1984, Société Radio-Canada, Centre d'archives GastonMiron, http://www.crlq.umontreal.ca/CAGM/rechsimple?query=159929.

+ Les belles heures, entrevue radiophonique avec Nicole Brossard réalisée par André Hamelin, animée par Suzanne Giguère et diffusée le 14 mai 1984, Société Radio-Canada, Centre d'archives Gaston-Miron, http://www.crlq.umontreal.ca/CAGM/rechsimple?query = 1565660.

+ DELEPOUlLE, Anne-Marie, «Entretien avec Nicole Brossard», Études canadiennes/Canadian Studies, $\mathrm{n}^{\circ} 16$, juin 1984, p. 67-71.

+ La fin des morales, émission radiophonique avec Nicole Brossard réalisée par Fernand Ouellette, animée par Paul Chamberland et diffusée le 21 novembre 1984, Société Radio-Canada, Centre d'archives Gaston-Miron, http://www.crlq.umontreal.ca/CAGM/rechsimple?query=697609.

+ GAUDET, Gérald, « Nicole Brossard. Toucher l'énergie», Voix d'écrivains. Entretiens, Montréal, Québec Amérique, coll. «Littérature d'Amérique», 1985, p. 215-223.

+ ROYER, Jean, «Entretien avec Nicole Brossard», Écrivains contemporains. Entretiens, t. III, Montréal, l'Hexagone, 1985, p. 163-169.

+ BONENFANT, Joseph et André GERVAIS, «Ce qui pouvait être, ici, une avant-garde. Entrevue avec Nicole Brossard, Roger Soublière et Marcel Saint-Pierre ", Voix et Images, vol. X, n 2, hiver 1985, p. 77-81.

+ ABEL, Marie-Christine, «La passion insolite de... Nicole Brossard: Le désert mauve», Le Devoir, 25 janvier 1986, p. 34.

+ Littératures, entrevue radiophonique avec Nicole Brossard réalisée par Gilbert Picard, animée par Claude Beausoleil et Michael Delisle et diffusée le 10 avril 1986, Société Radio-Canada, Centre d'archives Gaston-Miron, http://www.crlq.umontreal.ca/CAGM/rechsimple?query=1999526.

+ LOTBINIËRE-HARWOOD, Susanne de, «Translating Nicole Brossard. “Her Hand on a Book Resting While Our Bodies Obliquely" », Writing Magazine, octobre 1986, p. 36-41.

+ GAUVIN, Lise, «La question des journaux intimes. Table ronde», Études littéraires, vol. XXII, $\mathrm{n}^{\circ}$ 3, hiver 1987, p. 101-109.

+ CZACH, Elizabeth, «Feminization of the French Language», Otherwise, 24 mars 1987, p. 4.

+ En toutes lettres, entrevue radiophonique avec Nicole Brossard réalisée par Raymond Fafard, animée par Marie-Claire Girard et diffusée le 8 décembre 1987, Société Radio-Canada, Centre d'archives Gaston-Miron, http://www.crlq.umontreal.ca/CAGM/rechsimple?query=234202001.

+ NOTAR, Clea, «Interview with Nicole Brossard», Peter O’Brien (dir.), So to Speak. Interviews with Contemporary Canadian Writers, Montréal, Véhicule Press, 1988, p. 123-143.

+ Le jardin secret, émission radiophonique avec Nicole Brossard réalisée et animée par Gilles Archambault, diffusée le 13 janvier 1988, Société Radio-Canada, Centre d'archives GastonMiron, http://www.crlq.umontreal.ca/CAGM/rechsimple?query=709569.

+ YOKEN, Melvin B., «Nicole Brossard», Entretiens québécois, t. II, Montréal, Éditions Pierre Tisseyre, 1989, p. 109-131.

+ Dialogue, émission radiophonique avec Nicole Brossard réalisée par Fernand Ouellette, animée par Jean-Pierre Sauriol et diffusée le 27 août 1989, Société Radio-Canada, Centre d'archives Gaston-Miron, http://www.crlq.umontreal.ca/CAGM/rechsimple?query=712177.

+ JOUBERT, Ingrid, «Continent of Women. An Interview with Nicole Brossard», traduit du français par Jean Sourisseau, Prairie Fire, vol. X, n 3, 1989, p. 44-55.

+ GAUDET, Gérald, «La passion de la beauté. Entretien avec Nicole Brossard», Lettres québécoises, $\mathrm{n}^{\circ}$ 57, printemps 1990, p. 11-15. 
+ FIOCCHETTO, Rosanna, «Nicole Brossard. Parole per rinascere », Tuttestorie, nº 1, décembre 1990.

+ CBF bonjour, émission radiophonique avec Nicole Brossard réalisée par Micheline Richard, animée par Joël Le Bigot et diffusée le 15 mars 1991, Société Radio-Canada, Centre d'archives Gaston-Miron, http://www.crlq.umontreal.ca/CAGM/rechsimple?query=47717015.

+ Littératures actuelles, entrevue radiophonique avec Nicole Brossard réalisée par André Major, animée par Suzanne Giguère et diffusée le 21 avril 1991, Société Radio-Canada, Centre d'archives Gaston-Miron, http://www.crlq.umontreal.ca/CAGM/rechsimple?query=10954005.

+ POWELL, Marilyn, «The World According to Women. Part IV », Ideas, émission radiophonique réalisée et animée par Marilyn Powell, diffusée le 27 juin 1991, Canadian Broadcasting Corporation.

+ DAURIO, Berverley, "Order and Imagination », Books in Canada, vol. XX, n² 2, mars 1991, p. 1921.

+ FORTIER, Frances, «L'écriture "énigmatique" de Nicole Brossard», Nuit blanche, n 46, décembre 1991-janvier-février 1992, p. 36-41 (repris dans Nuit blanche, nº 69, hiver 1997, p. 84-87).

+ Ici comme ailleurs, émission radiophonique avec Nicole Brossard diffusée le 31 juillet 1991, Société Radio-Canada, Centre d'archives Gaston-Miron, http://www.crlq.umontreal.ca/CAGM/rechsimple?query=738588.

+ TREMBLAY, Odile, "L'adversaire du formalisme», Le Devoir, 12 novembre 1991, p. D1 et D2.

+ GONNARD, Catherine, «Entrevue avec Nicole Brossard», Lesbia, nº 120, 1993, p. 31-33.

+ VASSEUR, Annie Molin, «Entrevue avec Nicole Brossard», Arcade, n²8, 1993, p. 65-79.

+ WILLIAMSON, Janice Ray, «Before I Became a Feminist, I Suppose I Was an Angel, a Poet, a Revolutionary ", Sounding Differences. Conversations with Seventeen Canadian Women Writers, Toronto, University of Toronto Press, 1993, p. 57-74.

+ COOKE, Nathalie, «Energy, Emotion and Perspective. An Interview with Nicole Brossard», Arc, $\mathrm{n}^{\circ} 32,1994$, p. 55-61.

+ LARSON, Jacqueline et Jodey CASTRICANO, «Blue Period - That's a Story. A Conversation with Nicole Brossard and Daphne Marlatt», West Coast Line. A Journal of Contemporary Writing and Criticism, no 15, 1994-1995, p. 29-53.

+ HUFFER, Lynne, «An Interview with Nicole Brossard», Yale French Studies, n 87, 1995, p. $115-121$.

+ LAURIN, Danielle, «Subvertir, transgresser!», Le Devoir, 14 octobre 1995, p. D1.

+ MARTEL, Réginald, «L'écriture est un jeu, on y joue sa vie», La Presse, 19 novembre 1995, p. B7.

+ WACHTEL, Eleanor, «Interview with Nicole Brossard», More Writers \& Company, Toronto, Alfred A. Knopf, 1996, p. 286-301.

+ Qui êtes-vous?, entrevue radiophonique avec Nicole Brossard réalisée par André Major, animée par Normand de Bellefeuille et diffusée les 22, 23 et 24 janvier 1996, Société Radio-Canada, Centre d'archives Gaston-Miron, http://www.crlq.umontreal.ca/CAGM/rechsimple?query=734239.

+ CADORETTE, Johanne, « Nicole Brossard», Fugues, vol. XV, n 3, juin 1998, p. 82.

+ DAURIO, Beverley, «Patriarchal Mothers. Nicole Brossard», Beverley Daurio et Frank Davey (dir.), The Power to Bend Spoons. Interviews with Canadian Novelists, Toronto, Mercury, 1998, p. $42-48$.

+ CANTIN, David, «Comprendre est un séjour», Le Devoir, 9 octobre 1999, p. D6. 
+ DURAND, Marcella, «Interview with Nicole Brossard. On Translation and Other Such Pertinent Subjects », Double change, nº 2, 2001, http://www.doublechange.com/issue2/brossard. htm.

+ DURAND, Marcella, « "If I Am Really Myself”. On Translation (An Interview with Nicole Brossard)», Verdure, nos 5-6, 2002, p. 54-61.

+ RUSSO, Linda, «Sensational Intensities: Poetry and Prose. An Interview with Nicole Brossard», Verdure, $\mathrm{n}^{\text {os }}$ 5-6, 2002, p. 123-137.

+ CATELLIER, Maxime, « Nicole Brossard», Ici, semaine du 6 au 12 novembre 2003, p. VII.

+ SAINT-MARTIN, Lori, «La lucidité, l'émotion. Entretien avec Nicole Brossard, poète et romancière", Nouvelles questions féministes. Revue internationale francophone, vol. XXIII, nº 1 , 2004, p. 104-120.

+ FITZGERALD, Judith, "Nicole Brossard. Negotiating the Flash, the Burn », In Other Words. The Globe and Mail Blogs, 19 octobre 2009, http://www.theglobeandmail.com/blogs/in-otherwords/nicole-brossard-negotiating-the-flash-the-burn/article1329192/.

\section{G. Direction d'ouvrage collectif}

+ Le long poème, Québec, Nota bene, coll. «Cahiers du Centre Hector-De Saint-Denys-Garneau», 2011, $151 \mathrm{p}$.

\section{H. Textes radiophoniques}

+ Narrateur et personnages, dramatique inédite, Société Radio-Canada, 1971.

+ Lipstick et Juillet, œuvres radiophoniques réalisées par Gilbert Picard et diffusées le 12 novembre 1977, Société Radio-Canada, Centre d'archives Gaston-Miron, http://www.crlq.umontreal.ca/CAGM/rechsimple?query=648254.

+ Une impression de fiction dans le rétroviseur, Société Radio-Canada, 1978.

+ Journal intime, série d'émissions radiophoniques avec une lecture du journal intime de Nicole Brossard par Pol Pelletier réalisée par Yves Lapierre et diffusée du 8 au 12 août 1983, Société Radio-Canada, Centre d'archives Gaston-Miron, http://www.crlq.umontreal.ca/CAGM/ rechsimple?query=123938001.

+ La falaise, Société Radio-Canada, 1985.

\section{F i l m}

+ BROSSARD, Nicole, Luce GUILBEAULT et Margaret WESCOTT (réal.), Some American Feminists. New York 1976, Canada, ONF, 1977, 55 min (entrevues avec Kate Millett, Betty Friedan, TiGrace Atkinson et Rita Mae Brown).

\section{J. Participations à des documentaires}

+ LABRECQUE, Jean-Claude et Jean-Pierre MASSE (réal.), La nuit de la poésie 27 mars 1970, Canada, ONF, 1970, 111 min $18 \mathrm{~s}$.

+ LABRECQUE, Jean-Claude et Jean-Pierre MASSE (réal.), La nuit de la poésie 28 mars 1980, Canada, ONF, 1980, 115 min.

+ TODD HÉNAULT, Dorothy (réal.), Les terribles vivantes. Bersianik, Marchessault, Brossard, Canada, ONF, 1986, 84 min.

+ GUÉRIN, Dominique (réal.), Profession poète. Nicole Brossard, Canada, Productions Quatre Saisons, 1988, $30 \mathrm{~min}$. 
+ VERTUE, Suzanne (réal.), L'existence lesbienne raisonne, Canada, Réseau Vidé-Elle, 1988, 112 min (conférence de Nicole Brossard: «Mémoire. Hologramme du désir »).

+ ALLEYN, Nora (réal.), Fragments of a Conversation on Language, Canada, ONF, 1990, 19 min [avec Nicole Brossard, Louky Bersianik, Susan Crean, Dorothy Todd Hénaut, Jovette Marchessault, Daphne Marlatt, Gail Scott et Betsy Warland].

+ LABRECQUE, Jean-Claude et Jean-Pierre MASSE (réal.), La nuit de la poésie 1991, Canada, ONF, 1992, $59 \mathrm{~min}$.

+ COLBERT, Laurie et Dominique CARDONA (réal.), Thank God I'm a Lesbian, Canada, Kasandra Circus Film Foundation, 1992, 60 min [entrevues avec Nicole Brossard, Sarah Schulman, Dionne Brand, Julia Creet et Lee Pui Ming].

+ BARTH, Anne (réal.), La nuit verte du parc labyrinthe, scénario de Nicole Brossard, Canada, Productions La Sterne, 1993, 24 min.

+ BARTH, Anne et Diane TRÉPANIÈRE (réal.), L'aura des mots, Canada, Productions Vision-Top, 1996, 26 min [entrevues avec Nicole Brossard, Benoîte Groult, Jeanne Hyvart et Marie-Claire Blais].

+ SANTORO, Miléna et Jean-Pierre MASSE (réal.), La théorie, un dimanche. Sweet suite, Canada, Conifère Têtu, 2002, 85 min [avec Nicole Brossard, Louky Bersianik, Louise Cotnoir, Louise Dupré et France Théoret].

+ MAROTTE, Sylvain (réal.), Nicole Brossard, poète, série "Au fil des mots», La Prairie, Productions Téridan en collaboration avec la BAnQ, 2005, 25 min.

\section{K. A n thologies}

+ Les stratégies du réel/The Story So Far 6, Montréal/Toronto, La Nouvelle Barre du jour/Coach House Press, 1978, 341 p.

+ Anthologie de la poésie des femmes, en collaboration avec Lisette Girouard, Montréal, Les Éditions du remue-ménage, coll. «Connivences», 1991, 379 p.

+ Poèmes à dire la francophonie. 38 poètes contemporains, Bordeaux/Paris, Castor Astral/CNDP, 2002, $156 \mathrm{p}$

+ Baiser vertige. Prose et poésie gaies et lesbiennes au Québec, Montréal, TYPO, 2006, 340 p.

\section{Entrevues menées par Nicole Brossard}

+ "Téléphone. Paroles déguisées en textes ou interviews ", La Barre du jour, nºs 31-32, hiver 1972, p. 134-150 [comptes rendus d'entrevues menées par Nicole Brossard avec Jacques Brault, Jan Depocas, Mario Dumais, Charles Gagnon, Laurent Girouard, Gérald Godin, Jean-Marc Piotte, Jacques Renaud et Pierre Vallières].

+ Littérature au pluriel, entrevue radiophonique avec Hélène Cixous réalisée par Gilles Archambault, animée par Nicole Brossard et diffusée le 13 novembre 1974, Société RadioCanada, Centre d'archives Gaston-Miron, http://www.crlq.umontreal.ca/CAGM/rechsimple? query $=637177$.

+ «Une romancière et critique sur le phallocentrisme» [avec Hélène Cixous], Le Devoir, 30 novembre 1974, p. 16

+ Horizons, entrevue radiophonique avec André Beaudet et Joseph Bonenfant réalisée par Gilles Archambault, animée par Nicole Brossard et diffusée le 19 janvier 1975, Société Radio-Canada, Centre d'archives Gaston-Miron, http://www.crlq.umontreal.ca/CAGM/rechsimple?query= 649739. 


\section{Fonds d'a rchives}

+ Fonds Joseph Bonenfant [P54], Sherbrooke, Service des archives de l'Université de Sherbrooke, 2006 [correspondance de Nicole Brossard].

+ Fonds Nicole Brossard. 1965-2002, LMS-0244, Ottawa, Bibliothèque et archives du Canada, 2002.

\section{RÉCEPTION CRITIQUE ET PRINCIPALES ÉTUDES}

\section{A. Sur l'auteure et son ouve en général}

1. Livres, chapitres de livres

+ ANDERSEN, Marguerite, "Nicole Brossard, stratège de l'imaginaire», François Gallays, Sylvain Simard et Robert Vigneault (dir.), Le roman contemporain au Québec (1960-1985), t. VIII, SaintLaurent, Fides, coll. "Archives des lettres canadiennes», 1992, p. 171-183.

+ BAYARD, Caroline, "Nicole Brossard and Mallarmé's Shawl», The New Poetics in Canada and Québec. From Concretism to Post-Modernism, Toronto, University of Toronto Press, 1989, p. 183-190.

+ BEAUSOLEIL, Claude, «Nicole Brossard. La spirale ardente», Les livres parlent, Trois-Rivières, Écrits des Forges, coll. «Estacades», 1984, p. 54-63.

+ CARRIËRE, Marie, «Resurrecting the (M)Other. Nicole Brossard», Writing in the Feminine in French and English Canada. A Question of Ethics, Toronto, University of Toronto Press, 2002, p. 61-71.

+ COUILlARD, Marie et Francine DUMOUCHEL, «Symphonie féministe», Barbara Godard (dir.), Gynocritics. Feminist Approaches to Canadian and Quebec Women's Writing/Gynocritiques.

Démarches féministes à l'écriture des Canadiennes et des Québécoises, Toronto, ECW Press, 1987, p. 77-83.

+ COUILLARD, Marie, «La lesbienne selon Simone de Beauvoir et Nicole Brossard. Identité ou figure convergente?", Cécile Coderre et Marie-Blanche Tahon (dir.), Le deuxième sexe. Une relecture en trois temps, 1949-1971-1999, Montréal, Les Éditions du remue-ménage, 2001, p. 53-60.

+ COUILLARD, Marie, «Reinaldo Arenas et Nicole Brossard. Discours d'exclusion et discours d'inclusion», Daniel Castillo Durante et Patrick Imbert (dir.), L'interculturel au cœur des Amériques, Ottawa/Winnipeg, Université d'Ottawa/University of Manitoba, 2003, p. 121-132.

+ DRAPEAU, Renée-Berthe, Féminins singuliers. Pratiques d'écriture: Brossard, Théoret, Montréal, Triptyque, 1986, $118 \mathrm{p}$.

+ DUPRÉ, Louise, Stratégies du vertige. Trois poètes: Nicole Brossard, Madeleine Gagnon, France Théoret, Montréal, Les Éditions du remue-ménage, coll. «Itinéraires féministes », 1989, 265 p.

+ FLOTOW, Luise von, «Women's Desiring Voices from Quebec: Nicole Brossard, Anne Dandurand and Claire Dé. Us/Them: Translation », Gordon Collier (dir.), Transcription and Identity in PostColonial Literary Cultures, Amsterdam, Rodopi, 1992, p. 109-119.

+ FORSYTH, Louise, «Les numéros spéciaux de La (Nouvelle) Barre du jour. Lieux communs, lieux en recherche, lieu de rencontre», Suzanne Lamy et Irène Pagès (dir.), Féminité, subversion, écriture, Montréal, Les Éditions du remue-ménage, coll. «Itinéraires féministes», 1983, p. 175-184.

+ FORSYTH, Louise, «Beyond the Myths and Fictions of Traditionalism and Nationalism. The Political in the Work of Nicole Brossard», Paula Gilbert Lewis (dir.), Traditionalism, Nationalism and Feminism. Women Writers of Quebec, Wesport, Greenwood, 1985, p. 157-172.

+ FORSYTH, Louise, «Destructuring Formal Space/Accelerating Motion in the Work of Nicole Brossard ", Shirley Neuman et Smaro Kamboureli (dir.), A Mazing Space. Writing Canadian Women Writing, Edmonton, Longspoon/NeWest, 1986, p. 211-221. 
+ FORSYTH, Louise H., «Nicole Brossard and the Emergence of Feminist Literary Theory in Quebec Since $1970 »$, Barbara Godard (dir.), Gynocritics. Feminist Approaches to Canadian and Quebec Women's Writing/Gynocritiques. Démarches féministes à l'écriture des Canadiennes et Québécoises, Toronto, ECW Press, 1987, p. 211-221.

+ FORSYTH, Louise, «Fernand Ouellette et Nicole Brossard. La poésie à caractère spéculaire : deux moments, deux écritures», Cécile Cloutier et Ben Shek (dir.), La poésie de l'Hexagone. Évolution, signification, rayonnement, Montréal, l'Hexagone, 1990, p. 223-232.

+ FORSYTH, Louise H. (dir.), Nicole Brossard. Essays on Her Works, Toronto, Guernica, coll. "Writers series», 2005, 255 p.

+ FORSYTH, Louise, «La cité et les urbaines radicales dans l'œuvre de Nicole Brossard. Entrée au féminin dans la ville contemporaine», Marie-Blanche Tahon et Céline Widmer (dir.), Actes du 4 e Congrès international de recherches féministes dans la francophonie plurielle, t. 3 : Les femmes entre la ville et la cité, Montréal, Les Éditions du remue-ménage, 2007, p. 28-39.

+ GILBERT, Paula Ruth, Mary Jean GREEN et Karen GOULD, «Inscription of the Feminine. A Century of Women Writing in Quebec», Paula Ruth Gilbert, Mary Jean Green, Jane Moss, et Lee Briscoe Thompson (dir.), Women Writing in Quebec. Essays in Honor of Jeanne Kissner, Plattsburg, Center for the Study of Canada, Plattsburgh State University of New York, 2000, p. $12-40$.

+ GODARD, Barbara, "La Barre du jour. Vers une poétique féministe», Suzanne Lamy et Irène Pagès (dir.), Féminité, subversion, écriture, Montréal, Les Éditions du remue-ménage, coll. «Itinéraires féministes», 1983, p. 195-205.

+ GODARD, Barbara, «Theorizing Feminist Discourse/Translation», Susan Bassnett et André Lefebvre (dir.), Translation, History and Culture, London, Cassell, 1990, p. 87-96.

+ GOULD, Karen, «Writing and Reading "Otherwise”. Québec Women Writers and the Exploration of the Difference», Arnold E. Davidson (dir.), Studies on Canadian Literature. Introductory and Critical Essays, New York, Publications of the Modern Language Association of America, 1990, p. 207-225.

+ GOULD, Karen, «Nicole Brossard. Beyond Modernity or Writing in the Third Dimension", Writing in the Feminine. Feminism and Experimental Writing in Quebec, Carbondale, Southern Illinois University Press, 1990, p. 52-107.

+ GOULD, Karen, «Theory's Space in Recent Texts by Nicole Brossard and France Théoret», Raija Koski, Kathleen Kells et Louise Forsyth (dir.), Les discours féminins dans la littérature postmoderne au Québec, San Francisco, EMText, 1993, p. 127-141.

+ GOULD, Karen, «Rewriting "America". Violence, Postmodernity and Parody in the Fiction of Madeleine Monette, Nicole Brossard and Monique LaRue», Mary Jean Green, Karen Gould, Micheline Rice-Maximin, Jack A. Yeager et Keith L. Walker (dir.), Postcolonial Subjects, Francophone Women Writers, Minneapolis, University of Minnesota Press, 1996, p. 186-209.

+ GUILLEMETTE, Lucie, «Reduplication, traduction et palimpseste dans l'œuvre de Nicole Brossard. L'inscription d'un espace féminin ", Lucie Lequin et Catherine Mavrikakis (dir.), La francophonie sans frontières. Une nouvelle cartographie de l'imaginaire au féminin, Paris, L'Harmattan, 2001, p. 181-195.

+ HAECK, Philippe, «Traces d'une écrivante», La table d'écriture. Poéthique et modernité. Essais, Montréal, VLB éditeur, 1984, p. 163-170.

+ HUFFER, Lynne, «From Lesbos to Montreal. Brossard's Urban Fictions », Maternal Pasts, Feminist Futures, Stanford, Stanford University Press, 1988, p. 117-133. 
+ KALMAN NAVES, Elaine, «Nicole Brossard. The Writer as Explorer», The Writers of Montreal, Montréal, Véhicule Press, 1993, p. 185-189.

+ KNUTSON, Susan Lynne, Narrative in the Feminine. Daphne Marlatt and Nicole Brossard, Waterloo, Wilfrid Laurier University Press, 2000, 233 p.

+ LEBLANC, Julie, «Les postures déconstructionnistes de Nicole Brossard», Jean-Pierre Bertrand et Lise Gauvin (dir.), Littératures mineures en langue majeure. Québec/Wallonie-Bruxelles, actes du colloque international «Littératures mineures en langue majeure» tenu à Liège du 9 au 11 octobre 2001, Bruxelles/Montréal, P.I.E.-Peter Lang/Presses de l'Université de Montréal, coll. «Documents pour l'histoire des francophonies. Théorie», 2003, p. 113-122.

+ MATA BARREIRO, Carmen, « Nicole Brossard. Une écriture de passion, d'engagement et en recherche», Carmen Boustani (dir.), Aux frontières des deux genres. En hommage à Andrée Chedid, Paris, Éditions Karthala, 2003, p. 145-160.

+ MATA BARREIRO, Carmen, "Nicole Brossard. Le je en mouvement, l'exploration de l'intime», Denise Brassard et Evelyne Gagnon (dir.), Aux frontières de l'intime. Le sujet lyrique dans la poésie québécoise actuelle, Montréal, Université du Québec à Montréal, Figura. Centre de recherche sur le texte et l'imaginaire, coll. «Figura», 2007, p. 109-124.

+ MATA BARREIRO, Carmen, "Le je, le nous, l'utopie et la solidarité dans l'écriture et dans la lecture poétiques chez Nicole Brossard », Denise Brassard et Evelyne Gagnon (dir.), États de la présence. Les lieux d'inscription de la subjectivité dans la poésie québécoise actuelle, Montréal, XYZ éditeur, coll. «Théorie et littérature», 2010, p. 65-80.

+ MAUGUIĖRE, Bénédicte, "Nicole Brossard ou la "théorie-fiction" ", Traversée des idéologies et exploration des identités dans les écritures de femmes au Québec. 1970-1980, New York, Peter Lang, coll. «Francophone Cultures and Literatures», 1997, p. 275-288.

+ MILOT, Louise, «Nicole Brossard. Une influence coûteuse», Jacques Allard et Madeleine Frédéric (dir.), Modernité/Postmodernité du roman contemporain Québec-Acadie, actes du colloque international de Bruxelles tenu du 27 au 29 novembre 1985, Montréal, Université du Québec à Montréal, coll. «Les Cahiers du Département d'études littéraires », 1987, p. 77-86.

+ MOISAN, Clément, "Gwendolyn MacEwen - Nicole Brossard», Poésie des frontières. Étude comparée des poésies canadienne et québécoise, Montréal, HMH, coll. «Constantes», 1979, p. 224-250.

+ NEPVEU, Pierre, «Ontologie et écologie», L'écologie du réel. Mort et naissance de la littérature québécoise contemporaine, Montréal, Boréal, coll. «Papiers collés», 1988, p. 141-154.

+ PARKER, Alice, «Writing Against Writing and Other Disruptions in Recent French Lesbians Texts », Linda Kauffman (dir.), Feminism and Institutions. Dialogues on Feminist Theory, Cambridge, Basil Blackwell, 1989, p. 211-239.

+ PARKER, Alice, «Nicole Brossard. A Differential Equation of Lesbian Love», Karla Jay et Joanne Glasgow (dir.), Lesbian Texts and Contexts. Radical Revisions, New York, New York University Press, 1990, p. 304-329.

+ PARKER, Alice, "Under the Covers. A Synesthesia of Desire (Lesbian Translations)», Susan J. Wolfe et Julia Penelope (dir.), Sexual Practice, Textual Theory. Lesbian Cultural Criticism, Cambridge, Blackwell, 1993, p. 322-339.

+ PARKER, Alice, Liminal Visions of Nicole Brossard, New York, Peter Lang, coll. «Francophone Cultures and Literatures», 1998, 287 p.

+ PRIETO, René, «In-Fringe. The Role of French Criticism in the Fiction of Nicole Brossard and Severo Sarduy ", Gustavo Peres Firmat (dir.), Do the Americas Have a Common Literature?, Durham, Duke University Press, coll. «Interamericana», 1990, p. 266-281. 
+ ROSENFELD, Marthe, «The Development of a Lesbian Sensibility in the Work of Jovette Marchessault and Nicole Brossard », Paula Gilbert Lewis (dir.), Traditionalism, Nationalism and Feminism. Women Writers of Quebec, Westport, Greenwood Press, coll. «Contributions in Women's Studies», 1985, p. 227-239.

+ ROSENFELD, Marthe, "Modernity and Lesbian Identity in the Later Works of Nicole Brossard", Susan J. Wolfe et Julia Penelope (dir.), Sexual Practice, Textual Theory. Lesbian Cultural Criticism, Cambridge, Blackwell, 1993, p. 199-207.

+ RUDY, Susan, «I Know That All Has Not Been Said. Nicole Brossard in English», Pauline Butling et Susan Rudy (dir.), Writing in Our Time. Canada's Radical Poetry in English (1957-2003), Waterloo, Wilfrid Laurier University Press, 2005, p. 79-88.

+ SAINT-MARTIN, Lori, «Nicole Brossard et Daphne Marlatt. La fascination de l'écriture», Contre-voix. Essais de critique au féminin, Québec, Nuit blanche éditeur, coll. «Essais critiques», 1997, p. 145-164.

+ SIEMERLING, Winfried, "The Visibility of the Utopian Form in the Work of Nicole Brossard», Discoveries of the Other. Alterity in the Work of Leonard Cohen, Hubert Aquin, Michael Ondaatje, and Nicole Brossard, Toronto, University of Toronto Press, coll. «Theory/Culture», 1994, p. 173204.

+ VERDUYN, Christl, «Lunatic Vistas? Contemporary Women's Writing in Quebec», Barbara Godard (dir.), Gynocritics. Feminist Approaches to Canadian and Quebec Women's Writing/Gynocritiques. Démarches féministes à l'écriture des Canadiennes et des Québécoises, Toronto, ECW Press, 1987, p. 71-75.

+ VOLDENG, Evelyne, «Nicole Brossard ou la quête du corps générique», Lectures de l'imaginaire. Huit femmes poètes des deux cultures canadiennes (1940-1980), Valenciennes/Orléans, Presses universitaires de Valenciennes/Éditions David, 2000, p. 191-206.

+ WEIR, Lorraine, «From Picture to Hologram. Nicole Brossard's Grammar of Utopia», Shirley Neuman et Smaro Kamboureli (dir.), A Mazing Space. Writing Canadian Women Writing, Edmonton, Longspoon/NeWest, 1986, p. 345-352.

\section{Mémoires et thèses}

+ BLAIS, Joann Margaret, Nicole Brossard and Robert Kroetsch. Aesthetics and Poetic Projects, mémoire de maîtrise, University of Alberta, 1985, $141 \mathrm{f}$.

+ BOUDREAU, Douglas Leonard, "Louise Labé and Nicole Brossard: Je désire donc je suis», Conceiving the Nation. Literature and Nation-Building in Renaissance France and Post-Quiet Revolution Quebec, thèse de doctorat, Ohio State University, 1999, f. 157-208.

+ BOULANGER, Ghislaine, Les feintes de l'essentielle dans la théorie-fiction de Nicole Brossard, mémoire de maîtrise, Carleton University, 1994, 165 f.

+ BOULANGER, Ghislaine, Feintes, essences et mimésis chez Nicole Brossard, Patrick Imbert et Marie-Claire Blais, thèse de doctorat, Université d'Ottawa, 2004, 330 f.

+ CAMPBELL, Catherine, "Nicole Brossard. Our Last Chance at Silence», Hearing the Silence. A Legacy of Post-modernism, thèse de doctorat, Université de Sherbrooke, 2003, f. 106-151.

+ CARRIÈRE, Marie J., «Nicole Brossard. (M)Other to (M)other» et "Perfecting the Other», Poetics of the Other. Five Feminist Writers from English Canada and Québec, thèse de doctorat, University of Toronto, 1999, f. 71-90 et 255-267.

+ DANIS, Mariette, L'impossible réelle. Lecture partielle de l'œuvre de Nicole Brossard, mémoire de maîtrise, Université du Québec à Montréal, 1985, 115 f. 
+ DELEPOUlLE, Anne-Marie, La rage d'écrire ou le défi féminin dans l'œuvre de Nicole Brossard, thèse de doctorat, Université de Paris-Val de Marne, 1983, 448 f.

+ DEVAUX, Peggy, "Écriture féminine" and "terri-stories". The Intricate Links Between Space and Women's Writing in the Works of Nicole Brossard and Daphne Marlatt, mémoire de maîtrise, University of Calgary, 1997, $175 \mathrm{f}$.

+ DICKINSON, Peter Ernest, «Toward a Transnational, Translational Feminist Poetics. Lesbian Fiction/Theory in Canada and Québec », Here Is Queer. Nationalisms and Sexualities in Contemporary Canadian Literatures, thèse de doctorat, University of British Columbia, 1997, f. 226-269.

+ FLOTOW, Luise von, Quebec Feminist Writing. Integrating the Avant-Garde and the Political in the Works of Nicole Brossard and France Théoret, thèse de doctorat, University of Michigan, 1991, $135 \mathrm{f}$.

+ HALL, Kimberly Quinn, Multicultural Alliances and the Politics of Difference. Writing, Power, and Resistance, thèse de doctorat, State University of New York at Binghamton, 1994, $175 \mathrm{f}$.

+ HARUVI, Dina, L'émergence d'une avant-garde féministe et sa consécration au Québec. La BJ/NBJ de 1975 à 1985, thèse de doctorat, Université de Tel-Aviv, 2002, 389 f.

+ LAVIGNE, Nicole, Word Warriors. A Collective Journey of Canadian Translators: Driving the Stories of Nicole Brossard, 1970-2000, mémoire de maîtrise, York University, 2003, 101 f.

+ LONGUST, Bridgett Renée, «The Architectural Gesture of Writing. Christine de Pizan and Nicole Brossard ", Reconstructing Urban Space. Twentieth-Century Women Writers of French Expression, thèse de doctorat, University of Arizona, 1996, f. 145-201.

+ LUNDGREN, Jodi Margaret E., " "A Girl in Combat in the City of Men". The Civic, Resistant, Ontological Woman in the Work of Nicole Brossard», Narrative Aesthetics, Multicultural Politics, and (Trans)National Subjects. Contemporary Fictions of Canada, thèse de doctorat, University of Washington, 2001, f. 209-300.

+ MILlER, Bett, Dancing the Divide. Reading au féminin and the Work of Nicole Brossard, thèse de doctorat, University of California, 1993, $213 \mathrm{f}$.

+ MOYES, Lianne, " "Le spectacle de l'impensable". Nicole Brossard's Lesbian Writing Subject», Writing Subjects. Gertrude Stein, Djuna Barnes, Nicole Brossard, and Lola Lemire Tostevin, thèse de doctorat, York University, 1994, f. 172-234.

+ PAUL, Catherine Anne, Le processus de l'écriture d'un point de vue féministe. L'exemple de Nicole Brossard, mémoire de maîtrise, Queen's University, 1985, 55 f.

+ PELLETIER, Marc, «Le poème en prose au cœur de la modernité poétique. Nicole Brossard», De Silvio à Nicole Brossard. Le poème en prose dans la littérature québécoise, thèse de doctorat, Université d'Ottawa, 1987, f. 528-549.

+ QUIGLEY, Margaret Ellen, Desiring Intersubjects. Lesbian Poststructuralism in Writing by Nicole Brossard, Daphne Marlatt, and Dionne Brand, thèse de doctorat, University of Alberta, 2000, $485 \mathrm{f}$.

+ SAUCIER, Michèle, L'œil lové Volubilis. Les courbes d'une écriture. Lectures de/avec Nicole Brossard, mémoire de maîtrise, Université de Sherbrooke, 1981, 117 f.

+ TRAINOR, Kim, «Spiral(e) : Écriture au féminin/Writing in the Feminine», Feminist Poetics from "écriture féminine" to "The Pink Guitar", thèse de doctorat, Université McGill, 2004, f. 53-86.

+ VILLA, Elena M., «Dancing on the Horizon. Nicole Brossard's Poetics of Presence and the Feminine Sublime», Eloquent Flesh. Cross-cultural Figurations of the Dancer in Nineteenth- and Twentieth-Century Literature, thèse de doctorat, University of Oregon, 2006, f. 273-298. 


\section{Articles}

+ ANDERSEN, Marguerite, "Nicole Brossard: un imaginaire tonique», Resources for Feminist Research/Documentation sur la recherche féministe, vol. XV, nº 4, décembre 1986-janvier 1987, p. 22-24.

+ ANDERSEN, Marguerite, «Subversive Texts. Quebec Women Writers», Studies in Canadian Literature, vol. XIII, nº 2, 1988, p. 127-141.

+ ANDERSON, Kristine J., «Revealing the Body Bilingual. Quebec Feminists and Recent Translation Theory ", Studies in the Humanities, vol. XXII, nos 1-2, 1995, p. 65-75.

+ BAYARD, Caroline, "Subversion is the Order of the Day ", Essays on Canadian Writing, n ${ }^{\text {os }} 7-8$, automne 1977, p. 17-25.

+ BAYARD, Caroline, "Nicole Brossard et l'utopie du langage», Revue de l'Université d'Ottawa, vol. L, n 1 , janvier-mars 1980, p. 89-95.

+ BAYARD, Caroline, «Vingt ans pour la trajectoire d'une subversion. De Paul-Marie Lapointe à Nicole Brossard », Études canadiennes/Canadian Studies, n 12, juin 1982, p. 135-143.

+ BAYARD, Caroline, «Diff-errance, dissémination et subversion. Trois écrivaines féministes au Québec», North Dakota Quarterly, vol. LII, n 3, 1984, p. 79-86.

+ BEAUDET, André, " "Gynécophonie-s" suivi de "Dessins, obliques, profils" », La Nouvelle Barre du jour, nº 88, mars 1980, p. 111-132.

+ BERSIANIK, Louky, «Fieffée désirante», La Nouvelle Barre du jour, nos 118-119, novembre 1982, p. 99-112.

+ BONENFANT, Joseph, «Nicole Brossard, hauteur d'un texte», Voix et Images du pays, vol. IX, $\mathrm{n}^{\circ} 1,1975$, p. 223-235.

+ BRULOTTE, Gaëtan, «L'écriture priapique», Champs d'application, n 3, été-automne 1974, p. 4-17.

+ CARON, Pascal, «L'obsession du corps et la poésie québécoise. Une tentative d'ouverture théorique à partir des poèmes de Claude Beausoleil, Nicole Brossard et Michel Beaulieu ", Études littéraires, vol. XXXVIII, nº 1, automne 2006, p. 87-108.

+ CAUSSE, Michèle, «Sub-in-vertere», La Nouvelle Barre du jour, nos 118-119, novembre 1982, p. 147-149.

+ CHARRON, François, «Nicole Brossard, un écrivain classique», La Nouvelle Barre du jour, $\mathrm{n}^{\text {os }}$ 118-119, novembre 1982, p. 71-75.

+ CONLEY, Katharine, "Going for Baroque in the Twentieth Century. From Desnos to Brossard», Québec Studies, n 31, printemps-été 2001, p. 12-23.

+ COTNOIR, Louise, «Lecture tangentielle», La Nouvelle Barre du jour, nos 118-119, novembre 1982, p. 121-127.

+ CURRAN, Beverley, «"Je suis une Geisha devant mon ordinateur”. Nicole Brossard in Japanese Translation», Verdure, nos 5-6, 2002, p. 62-71.

+ DION, Robert, «Écrire ce qui donne à lire. Nicole Brossard, lectrice», Voix et Images, vol. XVII, $\mathrm{n}^{\circ}$ 2, hiver 1992, p. 250-263.

+ DUPRÉ, Louise, «Les utopies du réel», La Nouvelle Barre du jour, nºs 118-119, novembre 1982, p. 83-89.

+ DURANLEAU, Irène, «Le texte moderne et Nicole Brossard», Études littéraires, vol. XIV, nº 1, avril 1981, p. 105-122.

+ ENGELBRECHT, Penelope, «"Lifting Belly Is a Language". The Postmodern Lesbian Subject», Feminist Studies, vol. XVI, nº 1, printemps 1990, p. 85-115. 
+ FISETTE, Jean, «Écrire pour le plaisir», Voix et Images du pays, vol. V, nº 1, automne 1979, p. 197-201.

+ FISETTE, Jean, «L'écrevisse et l'impossible. Gloses autour de deux textes de Nicole Brossard», Voix et Images, vol. XI, nº 1, automne 1985, p. 63-75.

+ FLOTOW, Luise von, «Legacies of Quebec Women's “Écriture au féminin”. Bilingual

Transformances, Translation Politicized, Subaltern Versions of the Text of the Street », Journal of Canadian Studies/Revue d'études canadiennes, vol. XXX, nº 4, hiver 1995-1996, p. 88-109.

+ FORSYTH, Louise, «The Novels of Nicole Brossard. An Active Voice», Room of One's Own, vol. IV, nº 1, 1978, p. 30-38.

+ FORSYTH, Louise, "The Radical Transformation of the Mother-Daughter Relationship in Some Women Writers of Quebec», Frontiers. A Journal of Women Studies, vol. VI, nos 1-2, printempsété 1981, p. 44-49.

+ FORSYTH, Louise, «Regards, reflets, reflux, réflexions. Exploration de l'œuvre de Nicole Brossard », La Nouvelle Barre du jour, nos 118-119, novembre 1982, p. 11-25.

+ FRÉMONT, Gabrielle, «Le féminisme à la NBJ. Un second souffle», Voix et Images, vol. X, nº 2, hiver 1985, p. 133-137.

+ GAUVIN, Lise, "Petit essai sur l'essai au féminin", Québec Studies, n 11, automne 1990-hiver 1991, p. 117-125.

+ GODARD, Barbara, "The Avant-Garde in Canada. Open Letter and La Barre du jour", Ellipse, $n^{\text {os }} 23-24,1979$, p. 98-113.

+ GODARD, Barbara, "Je est une autre". Nicole Brossard au Canada anglais», La Nouvelle Barre du jour, $\mathrm{n}^{\text {os }} 118-119$, novembre 1982, p. 150-155.

+ GODARD, Barbara, «Women Loving Women Writing: Nicole Brossard», Resources for Feminist Research/Documentation sur la recherche féministe, vol. XII, nº 1, mars 1983, p. 20-22.

+ GODARD, Barbara, "Producing Visibility for Lesbians. Nicole Brossard's Quantum Physics», English Studies in Canada, vol. XXI, nº 1, 1995, p. 125-137.

+ GOULD, Karen, «Female Tracings. Writing as Re-Vision in the Recent Works of Louky Bersianik, Madeleine Gagnon and Nicole Brossard", American Review of Canadian Studies, vol. XIII, n², 1983, p. 74-89.

+ GOULD, Karen, «Daphne Marlatt \& Nicole Brossard», Signature. A Journal of Theory and Canadian Literature, $\mathrm{n}^{\circ}$ 3, 1990, p. 28-43.

+ HAJDUKOWSKI-AHMED, Maroussia, «La sorcière dans le texte (québécois) au féminin ", The French Review, vol. LVIII, nº 2, décembre 1984, p. 260-268.

+ HOLBROOK, Susan, «Mauve Arrows and the Erotics of Translation», Essays on Canadian Writing, $\mathrm{n}^{\circ}$ 61, 1997, p. 232-241.

+ KNUTSON, Susan, Kathy MEZEI, Daphne MARLATT, Barbara GODARD et Gail SCOTT, "Vers-ions Con-verse. A Sequence of Translations», Tessera, vol. VI, printemps 1989, p. 16-23.

+ KNUTSON, Susan, «Daphne Marlatt and Nicole Brossard. Writing Metanarrative in the Feminine», Signature. A Journal of Theory and Canadian Literature, n 3, 1990, p. 28-43.

+ KNUTSON, Susan, «Nicole Brossard's Elegant International Play, in Canada», Terry Goldie, Carmen Lambert et Rowland Lorimer (dir.), Theoretical Discourse/Discours théoriques, Montréal, Association for Canadian Studies, 1994, p. 187-202.

+ KNUTSON, Susan, «Reading Nicole Brossard», Ellipse, n 53, 1995, p. 9-19.

+ KNUTSON, Susan, "Nouns, Pronouns, Verbs "At Eye Level”. Nicole Brossard's Jeu de mots \& the Representation of Critical Subjectivity», Verdure, nos 5-6, 2002, p. 112-122. 
+ LALIBERTÉ, Yves, «Deux recueils de poèmes ou Supprimer l'excentricité s'est [sic] s'abstenir», Incidences, vol. II-III, $\mathrm{n}^{\circ}$ 1, janvier-avril 1979, p. 77-97.

+ LAMY, Suzanne, «Glossaire pour Nicole», La Nouvelle Barre du jour, nos 118-119, novembre 1982, p. 63-70.

+ MATA BARREIRO, Carmen, «Nicole Brossard. Le regard, la voix, le corps ou comment le génie d'une femme devient une référence», Revue des lettres et de traduction, nº 6, 2000, p. 351-362.

+ MATA BARREIRO, Carmen, «Femmes d'images et de mots. Les images visuelles dans l'œuvre romanesque de Nicole Brossard, Baroque d'aube et Hier ", Revue des lettres et de traduction, $\mathrm{n}^{\circ}$ 8, 2002, p. 305-318.

+ MCPHERSON, Karen S., "Memory and Imagination in the Writings of Nicole Brossard», International Journal of Canadian Studies/Revue internationale d'études canadiennes, $\mathrm{n}^{\circ} 22$, 2000, p. 87-102.

+ MILOT, Louise, «Margaret Atwood et Nicole Brossard. La question de la représentation», Voix et Images, vol. XI, nº 1, automne 1985, p. 56-62.

+ MOISAN, Clément, «Écriture et errance dans les poésies de Gwendolyn MacEwen et Nicole Brossard», Revue canadienne de littérature comparée, vol. II, hiver 1975, p. 72-92.

+ MORGAN, Ceri, «Writing Heartlands and Nicole Brossard's Hier», British Journal of Canadian Studies, vol. 24, nº 2, 2011, p. 195-210.

+ MOYES, Lianne, "Composing in the Scent of Wood and Roses. Nicole Brossard's Intertextual Encounters with Djuna Barnes and Gertrude Stein », English Studies in Canada, vol. XXI, n² 2 1995, p. 216-225.

+ MOYES, Lianne, "Caught in Each Other's Dreams. Nicole Brossard's Portrait of Djuna Barnes», Verdure, nos 5-6, 2002, p. 91-99.

+ NEPVEU, Pierre, «Trois romans de Nicole Brossard. Une histoire au présent», Incidences, vol. IV, $\mathrm{n}^{\mathrm{os}} 2-3,1980$, p. 129-138.

+ NEPVEU, Pierre, «Nicole Brossard. Notes sur une écologie», La Nouvelle Barre du jour, nos 118 119, novembre 1982, p. 139-144.

+ NEPVEU, Pierre, «BJ/NBJ. Difficile modernité», Voix et Images, vol. X, nº 2, hiver 1985, p. 159-165.

+ PASSIOUR, André C., «Histoire, mythologie et sexe. Mythes et réalités des cultures gaies et lesbiennes», Fugues, vol. XIV, nº 6, septembre 1997, p. 56-60 (sur Nicole Brossard et MichelMarc Bouchard).

+ POTVIN, Claudine, «Pratiques utopistes et discours muséologique dans l'écriture de Nicole Brossard et de Cristina Peri Rossi ", Tangence, n 47, mars 1995, p. 43-55.

+ ROY, André, «La verge au beau tarif. La différenciation signifiante généralisée», La Nouvelle Barre du jour, nos 118-119, novembre 1982, p. 113-120.

+ SABOURIN, Claude, "Les numéros "femmes" de la BJ/NBJ. Pour une transformation des pratiques discursives», Voix et Images, vol. X, n 2, hiver 1985, p. 125-132.

+ SAINT-MARTIN, Lori, «Anne Hébert et Nicole Brossard. De la poésie à la prose », Études littéraires, no 3, juillet-septembre 1989, p. 67-77.

+ SEQUIN, Lucie, «Nicole Brossard. Les mots-étreintes», Canadian Women's Studies/Les cahiers de la femme, vol. I, no 3, printemps 1979, p. 56-59.

+ THÉORET, France, «Le sens de la formule», La Nouvelle Barre du jour, nos 118-119, novembre 1982, p. 77-82.

+ VASSEUR, François, «Soulever les peaux : les décoller/ées», La Nouvelle Barre du jour, nos 118 119, novembre 1982, p. 129-137. 
+ VERWAAYEN, Kimberly, «Region/Body: In? Of? And? Or? (Alter/Native) Separatism in the Politics of Nicole Brossard», Essays on Canadian Writing, n 61, 1997, p. 1-16.

+ VILLEMAIRE, Yolande, "Vénus est une jeune femme rouge toujours plus belle», La Nouvelle Barre du jour, $\mathrm{n}^{\text {os }}$ 118-119, novembre 1982, p. 45-61.

\section{B. Sur des ouvrages en particulier}

a) Amantes

+ GODARD, Barbara, «Preface», Nicole Brossard, Lovhers, traduit du français par Barbara Godard, Montréal, Guernica, coll. «Essential Poets Series», 1986, p. 7-12.

+ LAMY, Suzanne, «Les enfants uniques nés de père et de mère inconnus », Barbara Godard (dir.), Gynocritics. Feminist Approaches to Canadian and Quebec Women's Writing/Gynocritiques.

Démarches féministes à l'écriture des Canadiennes et des Québécoises, Toronto, ECW Press, 1987, p. 199-210.

\section{b) À tout regard}

+ NEPVEU, Pierre, «La voix inquiète», Nicole Brossard, À tout regard, Montréal, Bibliothèque québécoise, coll. «Littérature», 1989, p. 7-12.

\section{c) Au présent des veines}

+ FORSYTH, Louise, «Bursting Boundaries in the Vast Complication of Beauty. Transported by Nicole Brossard's Au présent des veines », Verdure, nos 5-6, 2002, p. 100-108.

\section{d) Baroque d'aube}

+ MCPHERSON, Karen S., "Writing the Present in Nicole Brossard's Baroque d'aube», American Review of Canadian Studies, vol. XXX, n 3, automne 2000, p. 361-383.

+ MOYES, Lianne, «Rien de sacré. Baroque d'aube de Nicole Brossard aux limites des discours lesbiens-féministes sur la sexualité », International Journal of Canadian Studies/Revue internationale d'études canadiennes, vol. XXI, printemps 2000, p. 35-64.

+ OPREA, Denisa-Adriana, «Nicole Brossard, Baroque d'aube. Sujets nomades: écriture, désir lesbien et postmoderne», Une poétique du personnage dans cinq romans québécois contemporains au féminin (1980-2000). Métaféminisme postmoderne, thèse de doctorat, Université Laval, 2008, f. 201-284.

+ PARKER, Alice, «Myth and Memory in Nicole Brossard's Baroque d'aube and Vertige de l'avantscène», Paula Ruth Gilbert et Roseanna Lewis Dufault (dir.), Doing Gender. Franco-Canadian Women Writers of the 1990s, Madison/London, Teaneck Fairleigh Dickinson University Press/Associated University Presses, 2001, p. 36-52.

\section{e) French Kiss}

+ EIBL, Doris G., «Ā propos de French Kiss de Nicole Brossard ou "d'(u)ne offensive de chats sauvages dans les plates-formes de la narration" ", Rotraud von Kulessa (dir.), Études féminines/Gender Studies: en littérature, en France et en Allemagne, actes du colloque organisé par le Frankreich-Zentrum de l'Université Albert-Ludwig de Fribourg le 6 et 7 décembre 2002, Fribourg, Frankreich-Zentrum, 2004, p. 61-71.

+ HAREL, Simon, "L'espace des mots», Le voleur de parcours. Identité et cosmopolitisme dans la littérature québécoise contemporaine, Montréal, Le Préambule, coll. «L'univers des discours», 1989, p. 245-256. 
+ LADOUCEUR, Thérèse, Une écriture de la modernité. Lecture analytique de French Kiss de Nicole Brossard, mémoire de maîtrise, Université Laval, 1983, 142 f.

+ LÉARD, Jean-Marcel, «Du sémantique au sémiotique en littérature. La modernité romanesque au Québec», Études littéraires, vol. XIV, nº 1, avril 1981, p. 17-60.

+ VILLEMAIRE, Yolande, «Le french kiss de la Vénus rouge», Cul-Q, nos 8-9, janvier 1976, p. 63-85.

\section{f ) $\mathrm{Hier}$}

+ LAILLOU SAVONA, Jeannelle, «Genre littéraire et genre sexué dans Hier de Nicole Brossard», Voix et Images, vol. XXIX, n 2, hiver 2004, p. 143-155.

\section{g) Installations: avec et sans pronoms}

+ PARKER, Alice, «Surviving in Another Tongue. Nicole Brossard's Installations », Verdure, ${ }^{\circ \mathrm{os}}$ 5-6, 2002, p. 44-53.

\section{h) Journal intime}

+ ELSLIGER, Lise Josée, «Nicole Brossard and the Marginal Voice», The Rise of the Female Voice in Canadian Autobiography, mémoire de maîtrise, University of New Brunswick, 1995, f. 84-96.

+ HAVERCROFT, Barbara, «Hétérogénéité énonciative et renouvellement du genre. Le Journal intime de Nicole Brossard», Voix et Images, vol. XXII, n 1, automne 1996, p. 22-37.

+ LEBLANC, Julie, «Langage de l'espace et sexuation dans les journaux de Nicole Brossard», Louise Dupré, Jaap Lintvelt et Janet Paterson (dir.), Sexuation, espace, écriture. La littérature québécoise en transformation, Québec, Nota bene, coll. «Littérature(s)», 2002, p. 67-91.

+ LEBLANC, Julie, «Écriture et réécriture au féminin. Les journaux de Nicole Brossard», Genèse de soi. L'écriture du sujet féminin dans quelques journaux d'écrivaine, postface de Roland Le Huenen, Montréal, Les Éditions du remue-ménage, 2008, p. 45-70.

\section{i) La lettre aérienne}

+ FORSYTH, Louise, "Errant and Air-Born in the City», The Aerial Letter, traduit du français par Marlene Wildeman, Toronto, Women's Press, 1988, p. 9-26.

+ JAGOSE, Annamarie, "Space, Skin, Spiral. The Aerial Letter and its Holographic Projection », Lesbian Utopics, New York, Routledge, 1994, p. 43-68.

+ TELLIER, Carolyne, Argumenter au féminin. Étude des stratégies discursives et énonciatives dans La lettre aérienne de Nicole Brossard, Entre raison et déraison de France Théoret et La bulle d'encre de Suzanne Jacob, mémoire de maîtrise, Université de Sherbrooke, 2003, 205 f.

\section{j) L'amèr ou le chapitre effrité}

+ BOK, Christian, «I am Mother/I am Other. L'amèr and the Matter of Mater », Studies in Canadian Literature, vol. XVI, nº 2, 1991, p. 17-38.

+ BUNCEL, Penny, "L'enclos maternel, site de transformation du langage. L'amèr ou Le chapitre effrité de Nicole Brossard", Textual Studies in Canada/Études textuelles au Canada, vol. VII, 1995, p. 16-20.

+ COUPAL, Sophie, "Nicole Brossard et L'amèr ou Le chapitre effrité», Discours métalinguistique et pratiques d'écriture féministes, mémoire de maîtrise, Université McGill, 2000, f. 44-70.

+ DUPRÉ, Louise, «Du propre au figuré. Préface», Nicole Brossard, L'amèr ou Le chapitre effrité. Théorie/fiction, Montréal, l'Hexagone, coll. «Typo», 1988, p. 7-13. 
+ FORSYTH, Louise, «L'écriture au féminin. L'Euguélionne de Louky Bersianik, L'absent aigu de Geneviève Amyot, L'amèr de Nicole Brossard ", Journal of Canadian fiction, nos 25-26, 1979, p. 199-211.

+ GODARD, Barbara, "Preface», Nicole Brossard, These Our Mothers, or, The Disintegrating Chapter, traduit du français par Barbara Godard, Toronto, Coach House Press, 1983, p. 7.

+ GODARD, Barbara, «L'amèr or The Exploding Chapter. Nicole Brossard at the Site of Feminist Deconstruction", Atlantis. A Women's Studies Journal/Journal d'études sur la femme, vol. IX, $\mathrm{n}^{\circ} 2$, printemps 1984, p. 23-34.

+ LABERGE, Christian, "L'amèr de Nicole Brossard. L'écriture du corps et le corps de l'écriture », Protée, vol. XI, n² 2, été 1983, p. 120-124.

+ MOYES, Lianne, "Mothertongue". A Comparative Study of Nicole Brossard's L'amèr and Daphne Marlatt's How Hug a Stone, mémoire de maîtrise, University of Western Ontario, 1987, $121 \mathrm{f}$.

+ SANTORO, Miléna, "Drawing the Line and Transgressing Limits. Nicole Brossard's L'amèr», The Feminist Avant-Garde Text in France and Québec. A Study of Contemporary Fiction by Hélène Cixous, Nicole Brossard, and Jeanne Hyvrard, thèse de doctorat, Princeton University, 1994, f. 105-177.

+ SANTORO, Miléna, "Drawing the Line and Transgressing Limits. Nicole Brossard's L'amèr », Mothers of Invention. Feminist Authors and Experimental Fiction in France and Quebec, Montréal, McGill-Queen's University Press, 2002, p. 153-207.

+ SAUCIER, Michèle, "Lectures intimes. L'amèr, Le sens apparent, Amantes », La Nouvelle Barre du jour, $\mathrm{n}^{\text {os }}$ 118-119, novembre 1982, p. 27-43.

\section{k) Langues obscures}

+ DUPRÉ, Louise, «Espaces de la mémoire, espaces du féminin. Langues obscures de Nicole Brossard », Jeannette den Toonder (dir.), Voix du temps et de l'espace, Québec, Nota bene, coll. «Convergences», 2007, p. 347-363.

\section{1) Le désert mauve}

+ ABRAHAM, Sylvie Glanzberg, Sous couvert de couverture. Un angle de vision du paratexte de couvertures, thèse de doctorat, Queen's University, 2000, $255 \mathrm{f}$.

+ ARROYAS, Frédérique, «Principes d'engendrement et de structuration dans Le désert mauve de Nicole Brossard», Thérèse Michel-Mansour, Elizabeth Lasserre et Daniel Halliday (dir.), Le nondit dans la littérature, Toronto, Éditions GFA, 1991, p. 12-22.

+ BACHAND, Denis, «Du roman au cédérom. Le désert mauve de Nicole Brossard», Michel Larouche (dir.), Cinéma et littérature au Québec. Rencontres médiatiques, Montréal, XYZ éditeur, coll. «Documents», 2003, p. 43-53.

+ CHASSAY, Jean-François, «Érosion, Le désert mauve de Nicole Brossard», Spirale, nº 76, février 1988, p. 3.

+ CONLEY, Katharine, "The Spiral as Moebius Strip. Inside/Outside Le désert mauve», Québec Studies, no 18, 1994, p. 149-158.

+ CÔTÉ, Nicole, «Le désert mauve, un bildungsroman queer? ", French Literature Series, vol. XXXI, 2004, p. 135-148.

+ CURRAN, Beverley et Mitoko HIRABAYASHI, «Translation. Making Space for a New Narrative in Le désert mauve», International Journal of Canadian Studies/Revue internationale d'études canadiennes, nº 15, printemps 1997, p. 109-120. 
+ CURRAN, Beverley, «Re-reading the Desert in Hypertranslation», Stephen Tchudi (dir.), Western Future. Perspectives of the Humanities Committee at the Millennium, Reno, Nevada Humanities Committee, 2000, p. 245-259.

+ CURRAN, Beverley, "Reading Us Into the Page Ahead. Translation as a Narrative Strategy in Daphne Marlatt's Ana Historic and Nicole Brossard's Le désert mauve», Lieven D’Hulst et John Milton (dir.), Reconstructing Cultural Memory. Translations, Scripts, Literacy, Amsterdam, Rodopi, 2000, p. 165-178.

+ DION, Robert, «Traduction et interprétation: Le désert mauve», Le moment critique de la fiction. Les interprétations de la littérature que proposent les fictions québécoises contemporaines, Québec, Nuit blanche éditeur, coll. «Essais critiques», 1997, p. 61-68.

+ DUFFEY, Erin, The Wor(l)d of Women. Le désert mauve of Nicole Brossard, mémoire de maîtrise, University of Alabama, 1994, $61 \mathrm{f}$.

+ EL NOSSERY, Nevine, La problématique de la réécriture au féminin dans Le désert mauve de Nicole Brossard et Elle sera de jaspe et de corail de Werewere Liking, thèse de doctorat, Université de Montréal, 2000, 315 f.

+ FERRER, Carolina, «Traduction, fission et trahison. L'hologramme de J. Robert Oppenheimer dans Le désert mauve de Nicole Brossard», Jean-François Chassay, Anne Élaine Cliche et Bertrand Gervais (dir.), Des fins et des temps. Les limites de l'imaginaire, Montréal, Université du Québec à Montréal, Figura. Centre de recherche sur le texte et l'imaginaire, coll. «Figura», 2005, p. 115-143.

+ FOX, Chris, «Murder at the Red Arrow Motel. Nicole Brossard's Mauve Desert as Dystopic Mystery ", Atlantis. A Women's Studies Journal/Journal d'études sur la femme, vol. XXIII, nº 1 , automne 1998, p. 112-119.

+ GIACOPPE, Monika, «The task of the translator in Garcia Marquez's One Hundred Years of Solitude and Brossard's Mauve Desert», Bucknell Review, vol. XLVII, nº 1, 2004, p. 124-138.

+ GILLESPIE, Nancy Elizabeth, Non/Species of Gothic. Opening the Wound and Embracing the Phantom Limb in Nicole Brossard's Mauve Desert and Mary Shelley's The Mortal Immortal, The Parvenue, and Transformation, mémoire de maîtrise, Simon Fraser University, 2001, 123 f.

+ GOULD, Karen, «Féminisme, postmodernité, esthétique de lecture. Le désert mauve de Nicole Brossard», Louise Milot et Jaap Lintvelt (dir.), Le roman québécois depuis 1960. Méthodes et analyses, Sainte-Foy, Presses de l'Université Laval, 1992, p. 195-213.

+ GUERTIN, Carolyn, «Adriene Jenik, Mauve Desert: A CD-ROM Translation», Other Voices. Journal of the Literary and Visual Arts, vol. XI, nº 2, 1998, p. 86-90.

+ HOLBROOK, Susan Leslie, "Desire and Delirium in Le désert mauve/Mauve Desert», A Poetics of Translation in Twentieth-Century Writing, thèse de doctorat, University of Calgary, 1997, f. 50-99.

+ HOLBROOK, Susan, «Delirium and Desire in Nicole Brossard's Le désert mauve», Differences, vol. XII, nº 2, été 2001, p. 70-85.

+ HOTTE, Lucie, «La partie et le tout. Fragmentation et union dans Le désert mauve», Littératures, $\mathrm{n}^{\circ} 11,1993$, p. 53-66.

+ HOTTE, Lucie, «Le même et l'autre. L'identité textuelle dans Le désert mauve de Nicole Brossard», Lucie Hotte (dir.), La problématique de l'identité dans la littérature francophone du Canada et d'ailleurs, actes du colloque organisé par les étudiants du Département des lettres françaises de l'Université d'Ottawa les 12 et 13 mai 1994, Ottawa, Le Nordir, 1994, p. 144-150.

+ HOTTE-PILON, Lucie, Entre la réalité et la fiction. Structure et fonctionnement du Désert mauve de Nicole Brossard, mémoire de maîtrise, Université d'Ottawa, 1991, 123 f. 
+ IGBOEMEKA, Adeze, «La lecture, l'imaginaire et la relation à l'autre. Le désert mauve de Nicole Brossard», Margot Irvine, Daniel Halliday et Adeze Igboemeka (dir.), La relation, actes du colloque tenu à l'Université de Toronto les 14 et 15 mai 1994, Toronto, Éditions GFA, 1996, p. 56-64.

+ KNOX, Katie, «Désir dans Le désert mauve», Nicole Fortin et Jean Morency (dir.), Littérature québécoise. Les nouvelles voix de la recherche, Montréal, Nuit blanche éditeur, coll. «Les Cahiers du Centre de recherche en littérature québécoise de l'Université Laval. Série “colloques" ", 1994, p. 69-83.

+ LEBLANC, Julie, «Théorie et pratique de l'image photographique. De l'analogie mimétique à la codification du "visuel-visible" », Texte, nos 21-22, 1997, p. 219-249.

+ LESSARD, Bruno, "The Cinemato (holo)graphy of Performance. Translation and Gender in Adriene Jenik's Mauve Desert: A CD-ROM Translation », Beyond the Cinema? Digital Adaptation, Media Ecology, and Performance in the CD-ROM Environment, thèse de doctorat, Université de Montréal, 2006, f. 82-129.

+ MCGAHAN, Susan, "Cleavages or Mauve Desert as a Post-Structuralist Mystery Novel. ReCovering the "Private" Investigative Subject», Tessera, vol. XIII, automne 1992, p. 101-118.

+ MCQUAIN, Taryn L., Rejet et transformation de la figure de la mère dans la littérature contemporaine des femmes au Québec, thèse de doctorat, University of Louisiana at Lafayette, 2006, $167 \mathrm{f}$.

+ MCPHERSON, Karen S., «Post(Modern) Script. D'une langue à l'autre or Speaking in Other Tongues: Le désert mauve», Incriminations. Guilty Women/Telling Stories, Princetown, Princetown University Press, 1994, p.155-178.

+ PARKER, Alice, «The Mauve Horizon of Nicole Brossard», Québec Studies, n 10, 1990, p. 107119.

+ PATERSON, Janet M., "Apocalypse ou renouvellement? Le désert mauve», Moments postmodernes dans le roman québécois, Ottawa, Presses de l'Université d'Ottawa, 1993, p. 109123.

+ PATERSON, Janet M., «Fast So Fast. Dérives identitaires dans Le désert mauve de Nicole Brossard», Jaap Lintvelt, Richard Saint-Gelais, Will Verhoeven et Catherine Raffi-Beroud (dir.), Roman contemporain et identité culturelle en Amérique du Nord, actes d'un colloque tenu à Groningue et organisé conjointement par l'Université de Groningue et le Centre de recherche en littérature québécoise de l’Université Laval, Québec, Nota bene, coll. «Littérature(s)», 1998, p. 45-57.

+ PERRY, Catherine, «L'imagination créatrice dans Le désert mauve. Transfigurations de la réalité dans le projet féministe", Voix et Images, vol. XIX, n 3, printemps 1994, p. 585-607.

+ PICARD, Anne-Marie, "Arrêt sur images. Identité et altérité dans Le désert mauve de Nicole Brossard et Rose Mélie Rose de Marie Redonnet», Dalhousie French Studies, n 32, automne 1995, p. 101-112.

+ POTVIN, Claudine, «Utopies amoureuses. Le désir piégé? », Joëlle Cauville et Metka Zupančič (dir.), Réécriture des mythes. L'utopie au féminin, Amsterdam, Rodopi, 1997, p. 201-217.

+ SANTORO, Miléna, «Feminist Translation. Writing and Transmission Among Women in Nicole Brossard's Le désert mauve and Madeleine Gagnon's Lueur ", Roseanna Dufault (dir.), Women by Women. The Treatment of Female Characters by Women Writers of Fiction in Quebec Since 1980, Madison, Fairleigh Dickinson University Press, 1997, p. 147-168.

+ SANTORO, Miléna, "Feminist Translation and Nicole Brossard's Le désert mauve», Paula Ruth Gilbert, Mary Jean Green, Jane Moss et Lee Briscoe Thompson (dir.), Women Writing in Quebec. 
Essays in Honor of Jeanne Kissner, Plattsburg, Center for the Study of Canada, Plattsburgh State University of New York, 2000, p. 123-134.

+ SCHWERDTNER, Karin, «Fever, Fever, Forever. La course à l'aventure au féminin dans Le désert mauve de Nicole Brossard", Jeanette den Toonder, Jaap Lintvelt et Jean Morency (dir.), Romans de la route et voyages identitaires, Québec, Nota bene, coll. «Terre américaine», 2006, p. 87-110.

+ SERVIN, Henri, «Le désert mauve de Nicole Brossard, ou l'indicible référent », Québec Studies, $\mathrm{n}^{\circ}$ 13, automne 1991-printemps 1992, p. 55-63.

+ STURGESS, Charlotte, «Le désert mauve de Nicole Brossard. Polysémie de l'écriture, engagement politique, voyage au féminin ", Études canadiennes/Canadian Studies, n 38 , juin 1995, p. 77-83.

+ STURGESS, Charlotte, «Body, Text and Subjectivity in Margaret Atwood's The Handmaid's Tale and Nicole Brossard's Le désert mauve», Études canadiennes/Canadian Studies, n 49, décembre 2000, p. 59-66.

\section{m) Le centre blanc}

+ GIROUX, Robert et Hélène DAME, «Les critères de poéticité dans l'histoire de la poésie québécoise (sémiotique littéraire)", Études littéraires, vol. XIV, nº 1, avril 1981, p. 123-163.

+ MICHON, Jacques, «Surréalisme et modernité», Études françaises, vol. XI, nº 2, mai 1975, p. 121-129.

\section{n) Le sens apparent}

+ BELLEMARE, Madeleine, «Le sens apparent. Nicole Brossard répond aux questions de Madeleine Bellemare», Nos livres, vol. XI, octobre 1980.

\section{o) $M a u v e$}

+ MARLATT, Daphne, «Translating Mauve : Reading Writing», Tessera, vol. VI, printemps 1989, p. 27-30.

\section{p) Picture Theory}

+ BOUCHARD, Laurence, Picture Theory ou les stratégies d'une écriture, mémoire de maîtrise, Université du Québec à Trois-Rivières, 1988, 134 f.

+ FORSYTH, Louise, «Préface», Nicole Brossard, Picture Theory, Montréal, l'Hexagone, coll. «Typo», 1989, p. 7-26.

+ FORSYTH, Louise, «Les jeux de la représentation dans Picture Theory de Nicole Brossard», Collectif, Mises en scène d'écrivains. Assia Djebar, Nicole Brossard, Madeleine Gagnon, France Théoret, Sainte-Foy/Grenoble, Le Griffon d'argile/Presses universitaires de Grenoble, 1993, p. 73-86.

+ GODARD, Barbara, «Preface», Nicole Brossard, Picture Theory, traduit du français par Barbara Godard, Montréal, Guernica, coll. «Prose», 1991, p. 7-11.

+ GODARD, Barbara, "A Translator's Journal», Sherry Simon (dir.), Culture in Transit. Translating the Literature of Quebec, Montréal, Véhicule Press, 1995, p. 69-82.

+ KNUTSON, Susan Lynne, Contested Knowing. Narratological Readings of Daphne Marlatt's How Hug a Stone and Nicole Brossard's Picture Theory, thèse de doctorat, University of British Columbia, 1989, $280 \mathrm{f}$. 
+ KNUTSON, Susan, «Protean Travelogue in Nicole Brossard's Picture Theory. Feminist Desire and Narrative Form ", Modern Language Studies, vol. XXVII, nos 3-4, automne-hiver 1997, p. 197211.

+ PAUL, Catherine Anne, La commutation de codes dans Picture Theory de Nicole Brossard et (_) de Michèle Causse, thèse de doctorat, Queen's University, 1993, 278 f.

+ POTVIN, Claudine, «Écrire (dans) la ville. La Metropolis au féminin», Tangence, nº 48, octobre 1995, p. 84-96.

+ THOMPSON, Dawn, «Re-Inventing the World. Calculating the Con/Volutional Integrals of Holography in Nicole Brossard's Picture Theory", Writing a Politics of Perception. Memory, Holography, and Women Writers in Canada, Toronto, University of Toronto Press, 2000, p. 1641.

\section{q) Sold-out. Étreinte-illustration}

+ BEAUDET, André, «Le récit rouge», Brèches, n 2, été-automne 1973, p. 59-70.

\section{r) Suite logique}

+ BELLEFEUILLE, Normand de, "Suite logique. Pour une grammaire de la différence», La Nouvelle Barre du jour, nos 118-119, novembre 1982, p. 91-98.

\section{s) Un livre}

+ BÉLANGER, Jean-Marc, Métatextualisation d'un point de vue auctorial sur l'écriture et la lecture dans Un livre de Nicole Brossard, mémoire de maîtrise, Université du Québec à TroisRivières/Université du Québec à Rimouski, 1992, 148 f.

+ STRACHAN, Fiona, Un livre de Nicole Brossard. Lecture fictive, lecture réelle, mémoire de maîtrise, Université de Montréal, 1982, 162 f. 